Two-dimensional laser collision-induced fluorescence measurements of plasma properties near an RF plasma cathode extraction aperture

This article has been downloaded from IOPscience. Please scroll down to see the full text article.

2012 Plasma Sources Sci. Technol. 21055030

(http://iopscience.iop.org/0963-0252/21/5/055030)

View the table of contents for this issue, or go to the journal homepage for more

Download details:

IP Address: 141.211.173.82

The article was downloaded on 25/06/2013 at 20:32

Please note that terms and conditions apply. 


\title{
Two-dimensional laser collision-induced fluorescence measurements of plasma properties near an RF plasma cathode extraction aperture
}

\author{
B R Weatherford ${ }^{1}$, E V Barnat ${ }^{2}$ and J E Foster ${ }^{1}$ \\ ${ }^{1}$ Nuclear Engineering and Radiological Sciences, University of Michigan, Ann Arbor, MI 48109, USA \\ 2 Sandia National Laboratories, Albuquerque, NM 87123-1423, USA \\ E-mail: brweathe@umich.edu,evbarna@sandia.gov and jefoster@umich.edu
}

Received 22 March 2012, in final form 22 August 2012

Published 3 October 2012

Online at stacks.iop.org/PSST/21/055030

\begin{abstract}
A dense plasma structure was observed to form near the extraction aperture of a helium RF plasma cathode. Laser collision-induced fluorescence was used to generate two-dimensional spatial maps of the electron density and the effective electron temperature within the structure over a range of operating conditions. The aperture plasma reached densities nearly an order of magnitude higher than the surrounding bulk plasma. The sharp spatial change in density at the plasma structure boundary suggests the presence of a double layer sheath. Higher temperature electrons were also observed at the periphery of the plasma structure. Variations in the observed plasma structure with extracted electron current were found to be consistent with reported low pressure anode spot behavior. Measurements of plasma density within and at the boundary of the structure, and the dependence of these on the current extracted across the external gap, are compared with calculations and discussed.
\end{abstract}

(Some figures may appear in colour only in the online journal)

$\begin{array}{ll}\text { Abbreviations } \\ \text { RF: } & \text { radio frequency } \\ \text { DC: } & \text { direct current } \\ \text { HCA: } & \text { hollow cathode assembly } \\ \text { LCIF: } & \text { laser collision-induced fluorescence } \\ \text { ECR: } & \text { electron cyclotron resonance } \\ \text { OPO: } & \text { optical parametric oscillator } \\ \text { OPA: } & \text { optical parametric amplifier } \\ \text { ICCD: } & \text { intensified charge-coupled device } \\ \text { CRM: } & \text { collisional radiative model } \\ \text { LIF: } & \text { laser-induced fluorescence } \\ \text { PIE: } & \text { plasma-induced emission } \\ \text { FWHM: } & \text { full width at half-maximum }\end{array}$

\section{Introduction}

Plasma cathodes represent a class of electron sources in which the electron current is extracted from the boundary of a low temperature plasma discharge [1]. For some applications, these devices have advantages over conventional thermionic sources. These include extended cathode life, relatively relaxed gas purity requirements, reactive gas compatibility, high-current densities, pulsed operation, and broad, uniform beam profiles [2]. An emerging application area of plasma cathodes is that of plasma generation for electric propulsion applications. Currently, plasma propulsion devices such as gridded ion thrusters utilize the HCA as the source of electrons. In an HCA, an annular, gas-fed thermionic emissive insert supplies electrons that generate a dense discharge plasma from which electrons are extracted [3,4]. Several varieties of emitterless plasma cathodes have been developed, including RF [5], microwave ECR [6-10], resonant microwave cavity [11] and helicon [12] discharges. In fact, emitterless plasma cathodes have been successfully flown in space as demonstrated on the Hayabusa spacecraft exploratory mission to the asteroid Itokawa [13].

Paramount to the understanding of plasma cathode behavior is the ability to describe the mechanism of electron 
extraction from the source discharge plasma. In order for electrons to be efficiently extracted and accelerated from a plasma boundary, the applied voltage on the extraction electrode must exceed the bulk plasma potential. As the extraction bias is increased and electrons are lost from the bulk plasma, the bulk plasma potential increases correspondingly. Electron acceleration occurs when the increasing anode bias overtakes the increasing plasma potential. Plasma cathodes consist of two components: a 'source' plasma discharge chamber and an electron extraction circuit. Plasma is generated in the source chamber and an external anode is biased positively relative to the chamber to extract electron current. The downstream end of the source chamber is often terminated by an aperture, to decouple the plasma potential from the anode bias and to regulate the pressure inside the chamber. Because of the interconnected nature of the anode bias and plasma potential, it has been suggested that the extraction aperture size should be on the order of a Debye length in radius, in order to stabilize the plasma potential during spikes in electron emission [1].

Other studies have shown that a plasma cathode can be operated in a global nonambipolar mode, where all electrons are efficiently lost through the aperture and all ions are lost to the cathode wall [12]. The conditions for global nonambipolar flow are dictated by the areas of bounding surfaces and the particle masses in the plasma, as discussed by Baalrud et al in [14], and reviewed here. Consider the situation of ambipolar flow to a single conducting surface in contact with a plasma, where the electron and ion fluxes lost to the surface are exactly balanced,

$$
0.25 e n \bar{c} \exp \left(-\frac{e V_{\mathrm{p}}}{T_{\mathrm{e}}}\right)=0.6 e n \sqrt{k_{\mathrm{B}} T_{\mathrm{e}} / M_{\mathrm{i}}} .
$$

Here, $e$ is the elementary charge, $n$ is the plasma density, $T_{\mathrm{e}}$ is the electron temperature, $V_{\mathrm{p}}$ is the plasma potential referenced to the surface, $k_{\mathrm{B}}$ is the Boltzmann constant and the quantity $\sqrt{k_{\mathrm{B}} T_{\mathrm{e}} / M_{\mathrm{i}}}$ is the Bohm speed. Let $\mu$ be defined as $\mu=\sqrt{2.3 M_{\mathrm{e}} / M_{\mathrm{i}}}$, where $M_{\mathrm{e}}$ and $M_{\mathrm{i}}$ are the masses of electrons and ions, respectively. In the single-electrode ambipolar system, the plasma potential is then determined from the product $-k_{\mathrm{B}} T_{\mathrm{e}} * \ln (\mu) / e$. In a plasma cathode, however, there are two loss areas for electrons and ions: the extraction aperture and the wall of the source chamber. The type of sheath at each boundary (and thus the type of particle confined at each boundary) is determined by the geometry of the chamber and aperture, and the quantity $\mu$ for the source plasma. The optimal configuration in the plasma cathode for efficient electron current extraction is that of global nonambipolar flow, in which the total extracted electron current through the aperture is balanced by ion current lost to the chamber walls. This condition is achieved within a range of ion and electron loss areas, namely, the ratio of wall area $A_{\mathrm{w}}$ to aperture area $A_{\text {ap }}$ must fall in the range of $\left.\mu<A_{\text {ap }} / A_{w}<[(0.6 / \mu)-1]^{-1}\right)$. Here, $A_{\mathrm{w}}$ is the cathode surface area and $A_{\mathrm{ap}}$ is the 'effective' anode area [14]. The constraints on plasma cathode geometry for potentialstabilized operation (dictated by the Debye length) and global nonambipolar flow (dictated by global device geometry) may not always be simultaneously feasible. Indeed, many plasma cathodes have not been designed to operate in the either the stabilized or nonambipolar modes.

The purpose of this work was to investigate the mechanism of electron extraction from a RF plasma cathode, in terms of the aperture geometry and operating conditions of the source. Specifically, it was observed that during electron extraction from an RF plasma source, a dense plasma structure formed just upstream of the extraction aperture. The aperture plasma displayed several similarities to anode spots (or anode 'fireballs') which have often been observed at the surface of positively biased electrodes immersed within a low temperature plasma [15-17]. However, the presence of anode spots in plasma cathodes is rarely mentioned in the literature. They have been extensively studied near immersed, positively biased disc electrodes, but there has been little detailed study of these structures existing far from anode surfaces. Additionally, most studies of anode spots in general have relied on waveforms and electrostatic probe measurements. While these studies are extremely useful in describing fundamental transient behaviors of anode spots, preferential 'draining' of electrons or ions by probes in the spot may perturb the structure, and additional noninvasive measurements of anode spot characteristics are warranted. As the presence of the aperture plasma structure acts as the actual interface between the extraction electrode and the bulk plasma in plasma cathodes, the relationship between the aperture plasma properties and the deliverable electron current have important implications for the optimization of these devices for electric propulsion. A detailed investigation of the interdependence of these properties is the focus of the work presented in this paper.

In this study, LCIF was used to investigate the effect of electron current extraction on the internal source plasma in a helium plasma cathode setup. LCIF has been used as a noninvasive means of measuring electron densities and temperatures [18-22], and the technique was recently extended to generate two-dimensional maps of these plasma properties by Barnat and Frederickson [23]. This latter variation of the technique was used to generate two-dimensional maps of plasma density and effective electron temperature in the region near the aperture of an RF plasma cathode. The trends in the critical bias for dense plasma formation, currentvoltage characteristic and plasma potentials were studied and compared with those in anode spots formed near anode surfaces. In the plasma cathode configuration, the outer boundary of an anode spot acts as an expanding surface which collects electrons from the bulk plasma and determines the amount of current that can be delivered through the aperture of a plasma cathode.

\section{Experimental setup}

\subsection{Plasma source}

A schematic of the setup used in this experiment is shown in figure 1. The RF plasma source consisted of a $50 \mathrm{~mm}$ glass tube surrounded by a helical coil. The glass tube was 


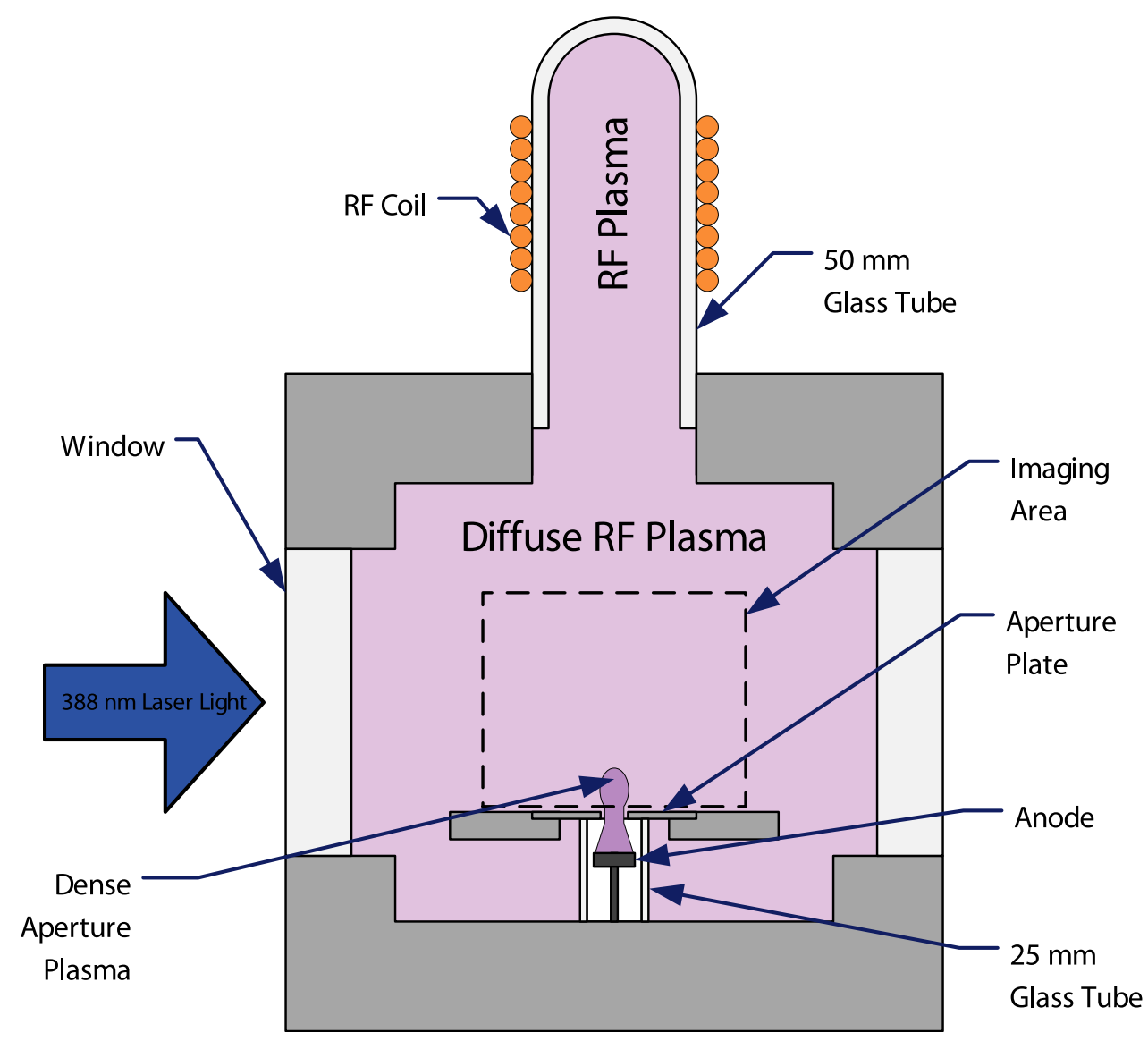

Figure 1. Schematic of plasma cathode setup.

mounted on the top face of a $150 \mathrm{~mm}$ (6 inch) stainless-steel cubic vacuum chamber. $100 \mathrm{~mm}$ below the downstream end of the RF source was a circular, grounded stainless-steel disc, $70 \mathrm{~mm}$ in diameter and $6 \mathrm{~mm}$ thick. The disc was bored through (roughly $25 \mathrm{~mm}$ diameter) in the center to accept smaller interchangeable discs $(1 \mathrm{~mm}$ thick), with varying aperture openings $(4.8 \mathrm{~mm}, 6.4 \mathrm{~mm}$ and $7.9 \mathrm{~mm}$ diameters $)$. An extraction anode, $12.7 \mathrm{~mm}$ in diameter, was mounted $38 \mathrm{~mm}$ below the aperture. A $25 \mathrm{~mm}$ glass tube was situated around the anode, between the grounded disc and the chamber wall. The anode was connected to a dc high voltage power supply, and the collected current was measured by the voltage drop across a $1 \Omega$ resistor. In this configuration, when a positive bias was applied to the anode, the system functionally behaved as a plasma cathode. The internal chamber walls and grounded disc acted as the cathode (ion collecting) surface, while the electrons were extracted from the RF plasma through the aperture.

Helium gas was fed into the chamber, and the internal pressure was controlled by a throttling valve. The regions inside the RF plasma source, chamber and anode-aperture gap are at the same neutral gas pressure. The pressures investigated in this study ranged from 75 to 200 mTorr. In all of the data presented here, the RF coil was driven at $22.1 \mathrm{MHz}$, which was chosen to minimize reflected power. The absorbed (forward minus reflected) RF power was fixed at $40 \mathrm{~W}$ in all cases, with typical reflected power levels of 10-15 W.

\subsection{LCIF diagnostic}

The LCIF diagnostic in this study is described in more detail by Barnat and Frederickson [23], and is summarized briefly here. The third harmonic of a pulsed Nd-YAG laser, at $355 \mathrm{~nm}$, was used to pump a tunable OPO system. The OPO was tuned to generate a $777 \mathrm{~nm}$ beam, which was frequency doubled, resulting in a $389 \mathrm{~nm}$ output beam. An 8-channel delay generator was used to trigger the laser pulse at $20 \mathrm{~Hz}$, with a pulse width of $10 \mathrm{~ns}$. With the use of neutral density filters, the laser pulse energy was reduced to a typical value of $0.5 \mathrm{~mJ}$. A series of lenses and a slit shaped the $389 \mathrm{~nm}$ light into a planar beam, $\sim 1 \mathrm{~mm}$ thick and $\sim 8 \mathrm{~cm}$ long, which passed through the plasma cathode setup, illuminating the imaging plane directly above the aperture. An ICCD camera was connected to the delay generator, which controlled the ICCD gate time and delay relative to the laser pulse. The ICCD was oriented perpendicular to the plane of the laser beam (that is, into the page in figure 1), and it was used to record images of the illuminated plane through interchangeable narrow-band filters. The delay generator and other ICCD parameters were controlled by computer interface. A digital oscilloscope (triggered by the delay generator) was used to monitor the ICCD gate pulses and the laser intensity via a fast photodiode. A schematic of the optical layout is shown in figure 2.

The $389 \mathrm{~nm}$ laser beam is tuned to the $\left(2^{3} \mathrm{~S} \rightarrow 3^{3} \mathrm{P}\right)$ transition in helium. Because the $\left(2^{3} \mathrm{~S}\right)$ state is metastable, 


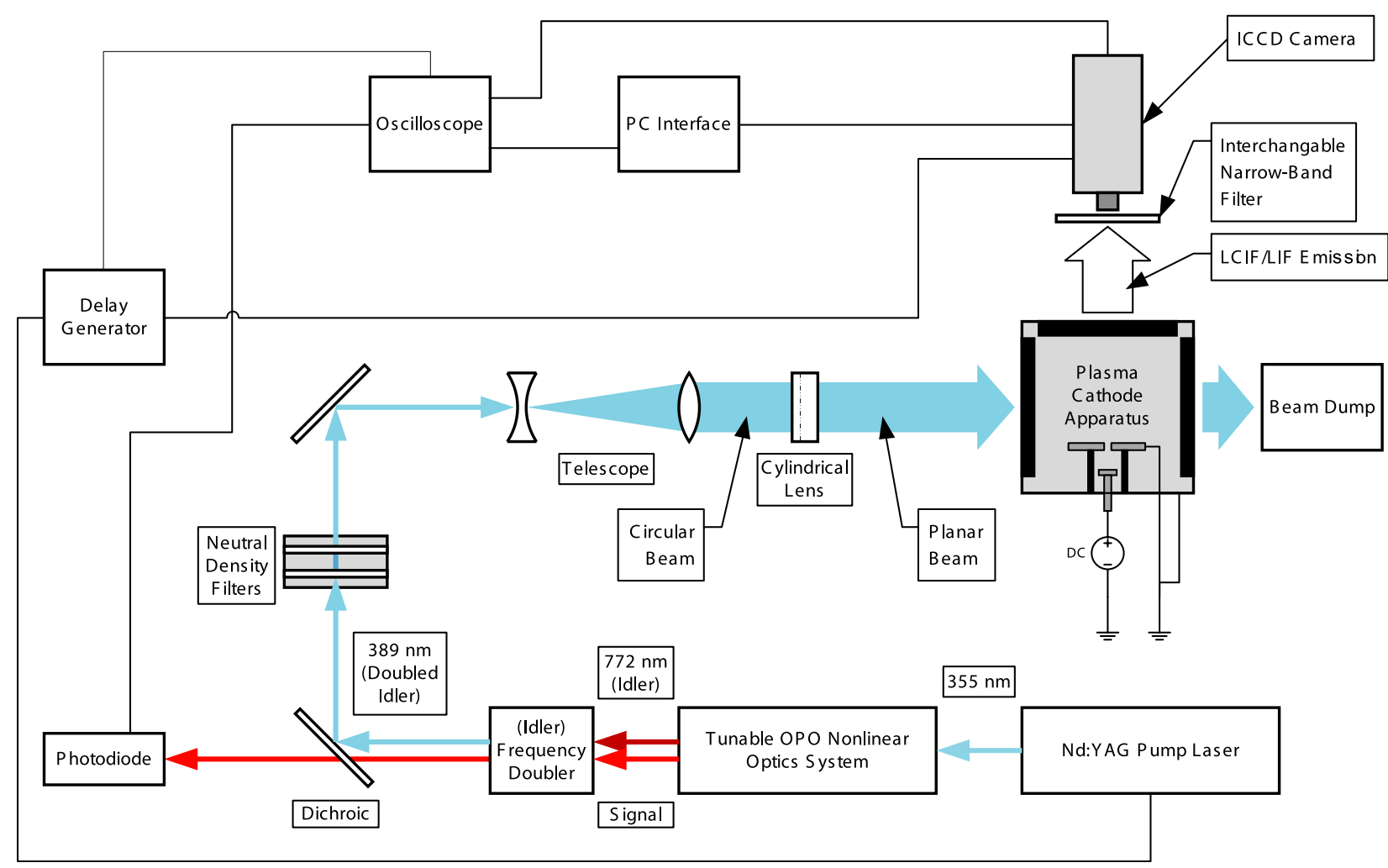

Figure 2. Schematic of controls and optical layout for the LCIF diagnostic. The ICCD camera is oriented out of the page, through the plasma cathode during experiments.

a reservoir of helium in that state is formed, and the laser beam pumps a fraction of the metastables to the $\left(3^{3} \mathrm{P}\right)$ state. Through collisions with the pumped neutrals, electrons will redistribute the population in the pumped state to nearby excited states, including the $\left(3^{3} \mathrm{D}\right)$ and $\left(4^{3} \mathrm{D}\right)$ states, among others. The time-integrated emission (after the laser pulse) from the $\left(3^{3} \mathrm{D} \rightarrow 2^{3} \mathrm{P}\right)(588 \mathrm{~nm})$ and $\left(4^{3} \mathrm{D} \rightarrow 2^{3} \mathrm{P}\right)(447 \mathrm{~nm})$ transitions, along with the $\left(3^{3} \mathrm{P} \rightarrow 2^{3} \mathrm{~S}\right)(389 \mathrm{~nm})$ transition, can be related to the electron density and temperature using a CRM.

The results from the CRM in [23] were used to determine the electron densities and temperatures from the intensities of the aforementioned transitions. In that study, a CRM was developed that modeled all spontaneous, electron-atom collision, and atom-atom collision processes between states in the helium triplet manifold, up to and including the $n=5$ states. The model was solved for a range of electron densities from $10^{9}$ to $10^{14} \mathrm{~cm}^{-3}$ and electron temperatures from $0.5-$ $6 \mathrm{eV}$. Under each plasma condition, evolution over time of the 389, 588 and $447 \mathrm{~nm}$ lines was calculated, from $50 \mathrm{~ns}$ before to $400 \mathrm{~ns}$ after the laser pulse. The intensities were then integrated over a $200 \mathrm{~ns}$ time period, starting $10 \mathrm{~ns}$ after the laser pulse. The ratio of the 588 to $389 \mathrm{~nm}$ integrated intensities were found to scale linearly with electron density, independent of electron temperature over the solution domain. The ratio of the $447-588 \mathrm{~nm}$ integrated intensities is sensitive to the electron temperature, and along with the electron density, this ratio can be used to uniquely determine an effective electron temperature.

After establishing the plasma conditions of interest, a narrow-band filter at $389 \mathrm{~nm}$ was installed in front of the ICCD.
Two series of images were recorded: one series during the integration window just after the laser pulse, and another series taken $1 \mathrm{~ms}$ after the laser pulse. The first series of images consisted of images containing LIF plus the background PIE, while the latter images contained the background PIE only. The same procedure was followed for the 588 and $447 \mathrm{~nm}$ LCIF transitions, by changing the interference filter for the desired transition. For each transition, the total number of images was varied by adjusting the total accumulation time (at 20 images per second). This was performed to maximize the imaged signal for each transition. During analysis, the PIE images were subtracted from the corresponding LIF and LCIF images, giving the emission from LIF and LCIF alone. The resulting series of images were then averaged to reduce noise, normalized to the accumulation time, and at each pixel the ratios of $588: 389 \mathrm{~nm}$ and $447: 588 \mathrm{~nm}$ intensities were calculated. At each pixel the electron density was calculated by interpolating the $588: 389 \mathrm{~nm}$ ratio in the CRM results. The density along with the $447: 588 \mathrm{~nm}$ ratio were interpolated (or linearly extrapolated) in the CRM to find the effective temperature.

The relative uncertainty in electron densities can be approximated from the three-level LCIF model explained by Dzierzȩga et al [21]. From that model, the ratio, $F_{j}$, of integrated LCIF intensity from state $j$ to the integrated LIF intensity is given by

$$
F_{j}=\frac{R_{j} A_{j} \epsilon_{j} \tau_{j} \lambda_{1}}{A_{i} \epsilon_{1} \lambda_{j}},
$$

where $A, \epsilon$ and $\lambda$, respectively, denote the spontaneous emission rate, detection efficiency and wavelength of each 
transition. Subscript 1 denotes the LIF transition from the pumped state and subscript $j$ denotes the measured LCIF transition from the collisionally populated state $j . R_{j}=$ $R_{j, \text { el }}+R_{j, \text { at }}$ is the population rate of state $j ; R_{j}$, el and $R_{j}$, at are the population rates due to electronic and atomic collisions, respectively. $\tau_{j}$ is the total depopulation rate of state $j$ due to spontaneous, electronic and atomic processes. The electron density $n_{\mathrm{e}}$ can then be written as

$$
n_{\mathrm{e}}=\frac{F_{j} A_{1} \epsilon_{1} \lambda_{j}}{A_{j} \epsilon_{j} \tau_{j} \lambda_{1} K_{j}}-\frac{R_{j, \text { at }}}{K_{j}},
$$

where $K_{j}$ is the rate constant for electron impact excitation from state 1 to state $j$. For the helium LCIF measurements of electron density in this experiment, states 1 and 2 correspond to the $3^{3} \mathrm{P}$ and $3^{3} \mathrm{D}$ states, respectively. Assuming an atomic rate constant of $3 \times 10^{-12} \mathrm{~cm}^{3} \mathrm{~s}^{-1}$ [24], with the most conservative bounds on plasma conditions observed in this study (200 mTorr and $n_{\mathrm{e}}=2 \times 10^{10} \mathrm{~cm}^{-3} \mathrm{~s}^{-1}$ ), the atomic population rate is $5 \%$ of the electronic rate, and the second term on the right-hand side of equation (3) can be neglected. The values of $A_{j}, \epsilon_{j}$ and $\lambda_{j}$ do not vary in space within a given imaging measurement, nor do they vary between individual images. The uncertainty introduced by the quantities $K_{j}$ and $\tau_{j}$ calculated in the model introduces only a systematic bias because the rate constant of the $3^{3} \mathrm{P} \rightarrow 3^{3} \mathrm{D}$ transition is insensitive to electron temperature. Therefore, the relative uncertainty in electron density in LCIF images is estimated as the relative fluctuation in the measured intensity ratios; $7 \%$, as determined by shot-toshot variations under the most unstable condition seen in this study. The systematic bias in the absolute value of electron density is dominated by the uncertainty in the rate constants $K_{j}$, which Ralchenko conservatively estimates as $50 \%$ [25]. This systematic uncertainty is consistent with the factor of 1.5 agreement between LCIF and double probe measurements observed by Barnat [23].

It should be noted that estimates of the electron temperature using LCIF rely on the assumption of a Maxwellian electron energy distribution when calculating the intensity ratios for a given temperature and density. Deviations from this assumption will complicate the quantitative determination of electron temperatures. This effect is most pronounced when there is a significant population of highenergy electrons, as in a drifting Maxwellian or beam-like distribution. The results from temperature-sensitive LCIF are therefore presented as 'effective' temperatures that correspond to the observed LCIF intensities for the idealized Maxwellian plasma. Measurements that show high effective electron temperatures $(>8 \mathrm{eV})$ are extrapolated from the CRM results, which introduces an additional estimated uncertainty of $\sim 20 \%$ at high values. Although 'effective temperatures' on the order of $8 \mathrm{eV}$ or more are unlikely to be temperatures in the formal sense, these results would still provide useful means for qualitatively capturing spatial variations in average electron energy.

\subsection{Langmuir probe}

For some tests, cylindrical Langmuir probes were used to characterize the bulk plasma and aperture plasma potentials.

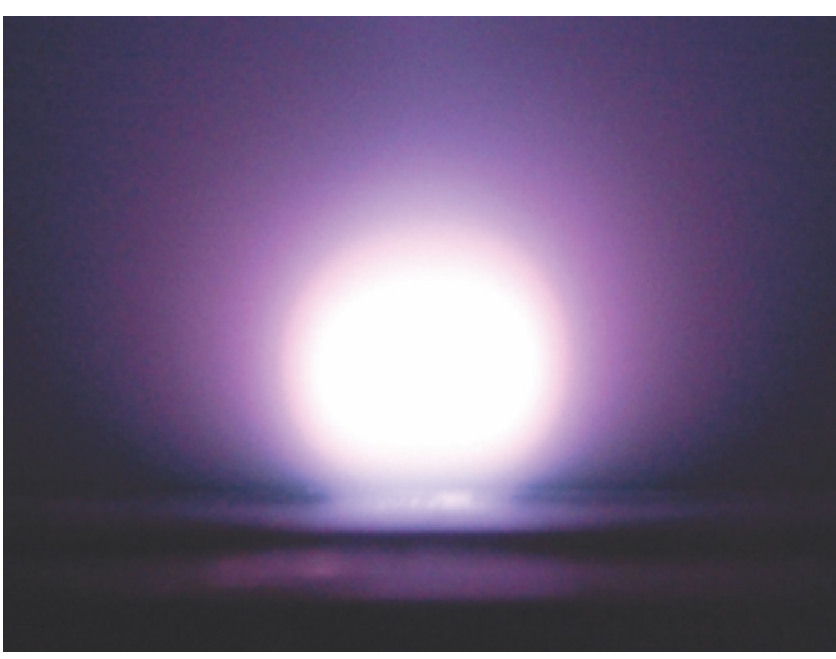

Figure 3. Photograph of an aperture plasma structure.

Both probes, made of tungsten, were $0.25 \mathrm{~mm}$ in diameter, centered above the aperture, and oriented parallel to the aperture plate. The bulk plasma probe was $7 \mathrm{~mm}$ long and located $25 \mathrm{~mm}$ above the extraction aperture. The aperture plasma probe was $2 \mathrm{~mm}$ long and located $3 \mathrm{~mm}$ above the aperture. The probe bias was applied by a $120 \mathrm{~V} \mathrm{DC}$ power supply, through a filtering circuit consisting of a band-stop filter and a low-pass filter. The $-3 \mathrm{~dB}$ attenuation frequencies of the band-stop filter were 19.6 and $27.7 \mathrm{MHz}$, with $-12 \mathrm{~dB}$ attenuation at $22 \mathrm{MHz}$; the low-pass filter cutoff frequency was $6 \mathrm{MHz}$. The probe bias and probe current during the sweep were recorded on an oscilloscope. The filtering circuit was tuned to minimize the magnitude of oscillations in the measured floating potential. After tuning, peak-topeak floating potential oscillations were less than $0.1 \mathrm{~V}$. As this peak-to-peak voltage oscillation is much less than the electron temperature, RF distortion of the probe trace can be neglected [26].

\section{Experimental results}

When the extraction anode was biased relative to the chamber wall, above a certain threshold voltage, the dense plasma structure was observed on the upstream side of the aperture; a photograph of an aperture plasma structure observed in a similar apparatus is shown in figure 3 [27]. Because the formation of the aperture plasma appeared to be required for increased plasma cathode electron emission, the plasma conditions within the structure were mapped using the LCIF technique.

It is worth mentioning here that in this study, there was no noticable influence of instabilities on the LCIF measurements of the plasma structure or the current-voltage characteristics. Instabilities have been observed in similar 'fireball' plasma structures at the surface of positively biased anodes, which have been thoroughly studied by Stenzel et al [28-31]. Instabilities can cause oscillations in the current and voltage waveforms at the anode, as well as extinction of the plasma structure. In high-current fireballs, when the ejection of ions 


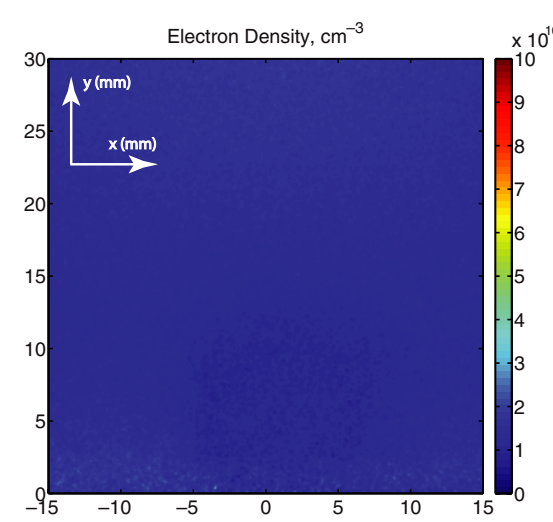

(a) $0 \mathrm{~mA}$

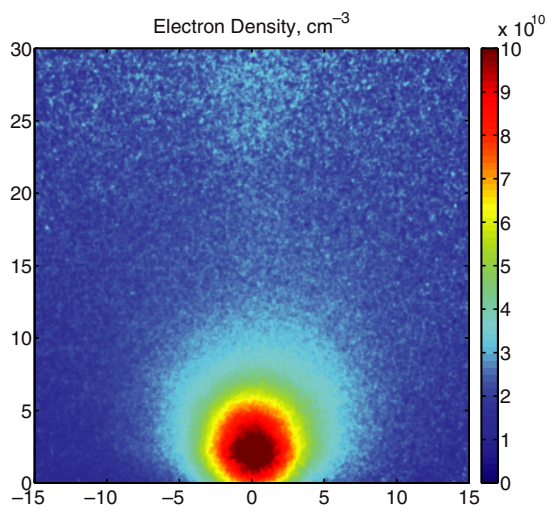

(d) $100 \mathrm{~mA}$

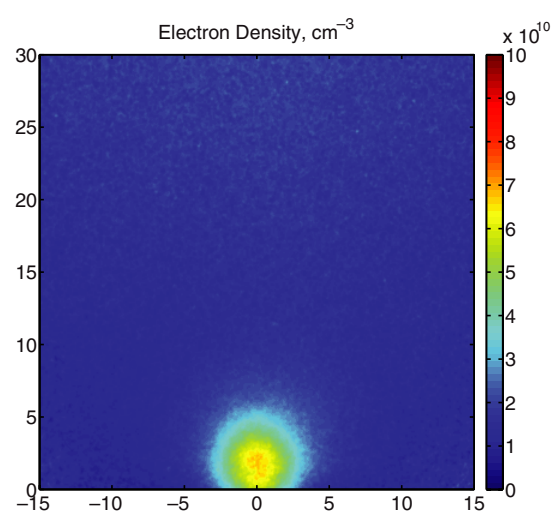

(b) $25 \mathrm{~mA}$

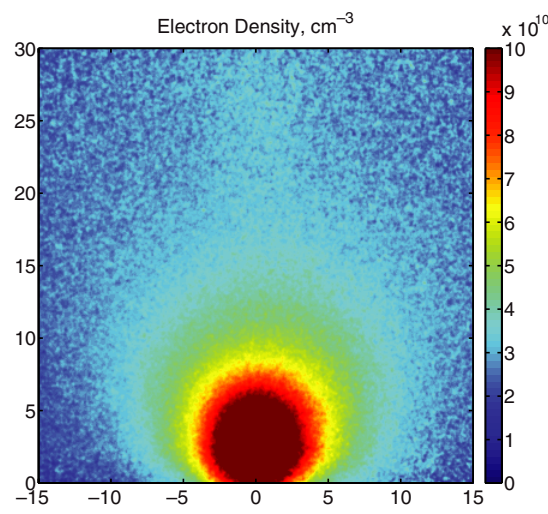

(e) $150 \mathrm{~mA}$

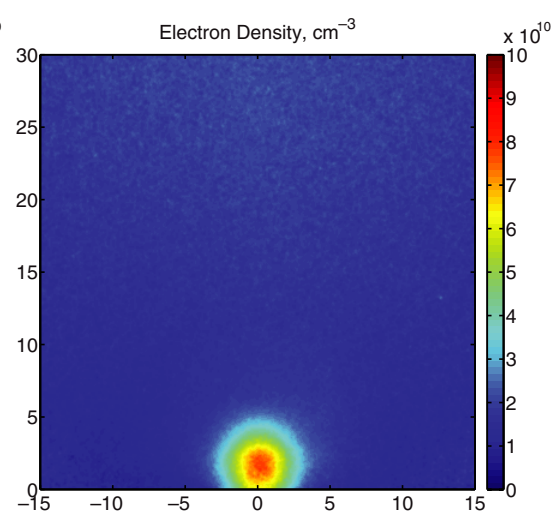

(c) $50 \mathrm{~mA}$

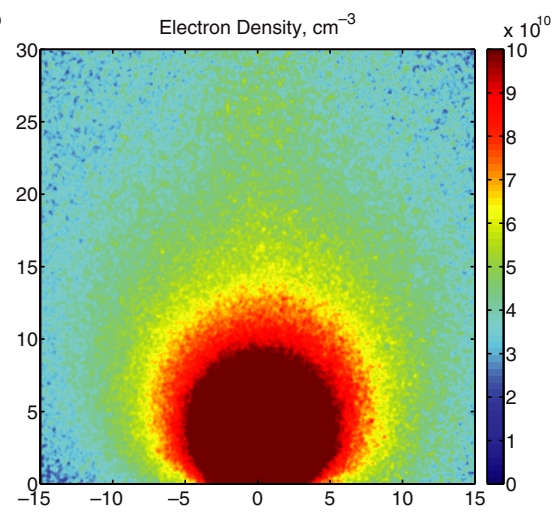

(f) $200 \mathrm{~mA}$

Figure 4. Maps of electron density with $6.4 \mathrm{~mm}$ aperture, $130 \mathrm{mTorr}$. Axis labels denote position, in mm. Average electron density in ( $a$ ) is $\sim 2 \times 10^{10} \mathrm{~cm}^{-3}$.

from the fireball overtake ion production within the fireball, the surrounding double layer collapses and the fireball decays [28]. Subsequent fireballs can form and decay at random times, and when fireballs are not present, electron-sheath instabilities can cause current oscillations [29]. During anode voltage pulses on the order of microseconds, overshooting current transients and sheath plasma oscillations are observed [30]. Also, ionization in the electron sheath near the anode surface can cause outward expansion of the sheath while partially neutralizing it; this decreases ionization in the sheath, thus driving an ionization instability [31].

In this work, the effects of electrode voltage transients were avoided by increasing the anode bias gradually and holding it constant for several seconds before taking measurements. As the images were taken, there were no significant variations in the size or intensity of the emission profiles between shots under fixed conditions. It will be shown from the steady-state current-voltage characteristics that the spot is sustained at lower voltages on the voltage downswing than on the upswing, suggesting that the spot is not extinguished via instabilities during the measurements. Although oscillations in the electron sheath may be present, the time-averaged properties of the aperture plasma structure (and the dependence of the steady-state extracted current on those properties) are quantities of interest with respect to plasma cathode configurations. The goal of the following measurements was to investigate these equilibrium properties.

\subsection{Variable current}

A series of electron density maps is shown in figure 4, for the plasma cathode operating at 130 mTorr on helium; the center of the aperture is located at coordinates ( $x=0 \mathrm{~mm}, y=0 \mathrm{~mm}$ ). Figure 4(a) depicts the measured electron density map when no extraction voltage is applied, and this is essentially an image of the RF-generated bulk plasma. The electron density in the bulk (with no applied bias) was around $2 \times 10^{10} \mathrm{~cm}^{-3}$ and quite uniform. When the aperture plasma structure is present, the plasma density maximum occurs a few millimeters upstream of the aperture. For example, the peak electron density for the $100 \mathrm{~mA}$ electron extraction case was $1.1 \times 10^{11} \mathrm{~cm}^{-3}$, at a distance of roughly $2 \mathrm{~mm}$ upstream of the aperture. In all cases, the electron density reaches its peak just upstream of the aperture, and in this sense, these density profiles are analogous to what has been seen in HCA and other plasma cathodes $[4,11,32]$.

A similar series of density maps is shown in figure 5 at an operating pressure of 200 mTorr. Like the 130 mTorr case, the structure also had a spherical shape, but at the elevated pressure, the diameter was smaller. The peak electron density was higher at the elevated pressure; at $100 \mathrm{~mA}$ and $200 \mathrm{mTorr}$, the peak electron density (which also occurred at roughly $2 \mathrm{~mm}$ upstream of the orifice) was roughly $30 \%$ higher than in the case with 130 mTorr. As the extracted current was increased, the density of the bulk above the aperture increased as well, even though this region is far outside the aperture plasma 


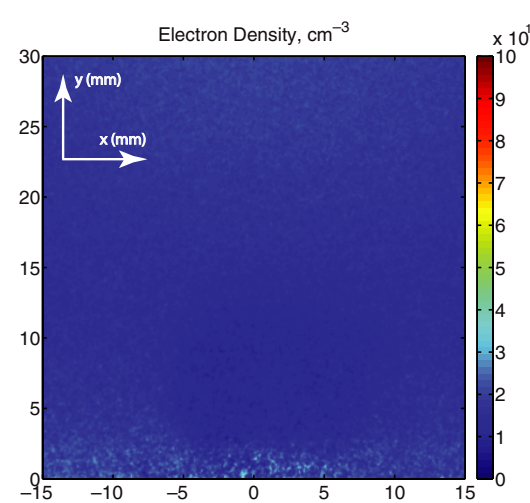

(a) $0 \mathrm{~mA}$

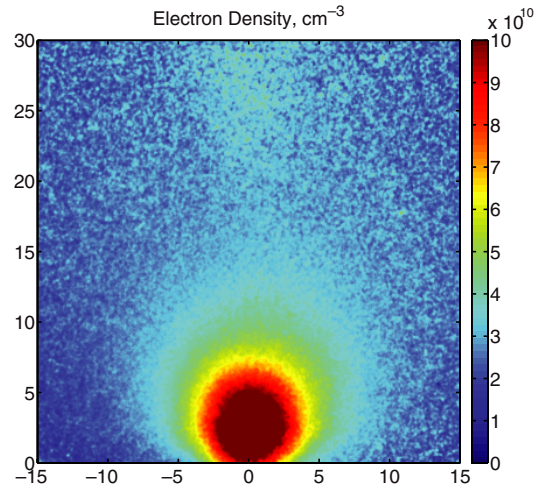

(d) $100 \mathrm{~mA}$

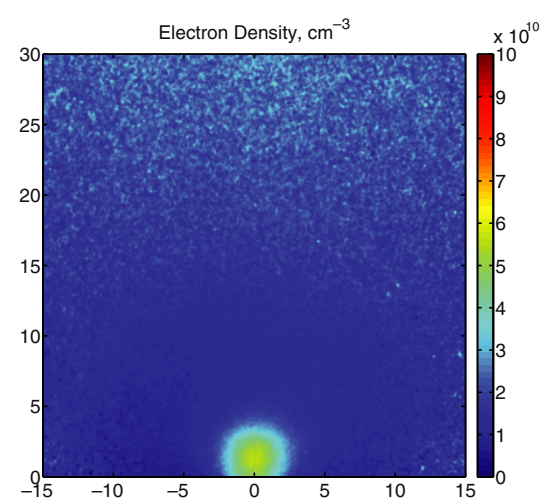

(b) $25 \mathrm{~mA}$

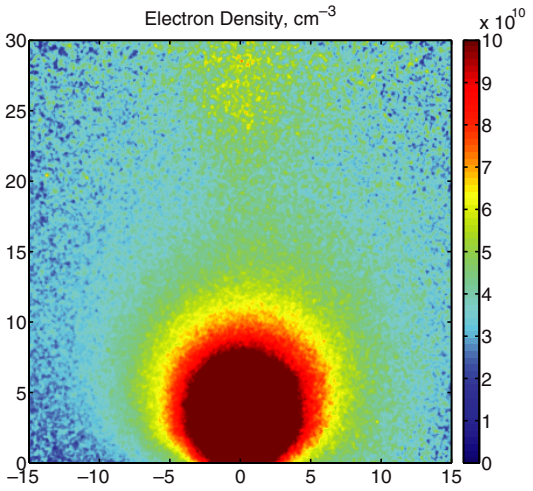

(e) $150 \mathrm{~mA}$

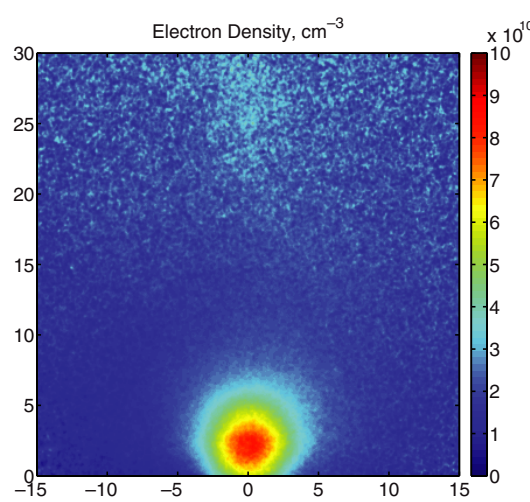

(c) $50 \mathrm{~mA}$

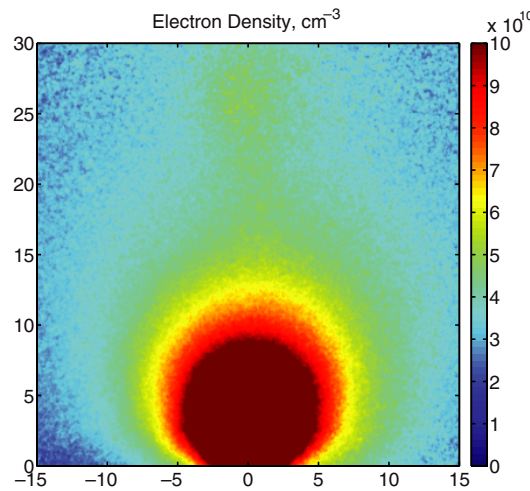

(f) $200 \mathrm{~mA}$

Figure 5. Maps of electron density with $6.4 \mathrm{~mm}$ aperture, $200 \mathrm{mTorr}$. Axis labels denote position, in mm. Average electron density in $(a)$ is $\sim 2 \times 10^{10} \mathrm{~cm}^{-3}$.

structure. The variation in electron density along the vertical axis, at 200 mTorr, is shown as a function of extracted current in figure 6. All of these profiles converged to the same bulk plasma density far upstream of the aperture, suggesting that the presence of the aperture plasma structure does not affect the upstream source plasma density under these conditions. The peak electron density occurred $3 \mathrm{~mm}$ from the aperture for all of the profiles in figure 6 with the exception of the $25 \mathrm{~mA}$ case. At $25 \mathrm{~mA}$, the aperture plasma had contracted in width and the peak density shifted to $2 \mathrm{~mm}$ from the aperture.

The spatial variation of the electron temperature in the aperture plasma region are shown in figures 7 and 8 . The measurements suggest the presence of a 'halo' region of high-energy electrons surrounding the inner core of the aperture plasma. The 'halo' is particularly noticeable in the temperature maps corresponding to lower extraction currents, as in figures $7(b)$ and $8(b)$. The thin, high-energy electron layer suggests the presence of an accelerating potential, most likely in the form of a double layer, surrounding the aperture plasma structure. The determination of the electron temperature in figures 7 and 8 is less straightforward than the determination of electron density. Again, the LCIF technique assumes a Maxwellian distribution for the electrons, which was incorporated in the calculation of energyaveraged rate constants. If an accelerating potential gradient existed between the aperture and bulk plasma regions, the electrons in the aperture plasma would significantly deviate

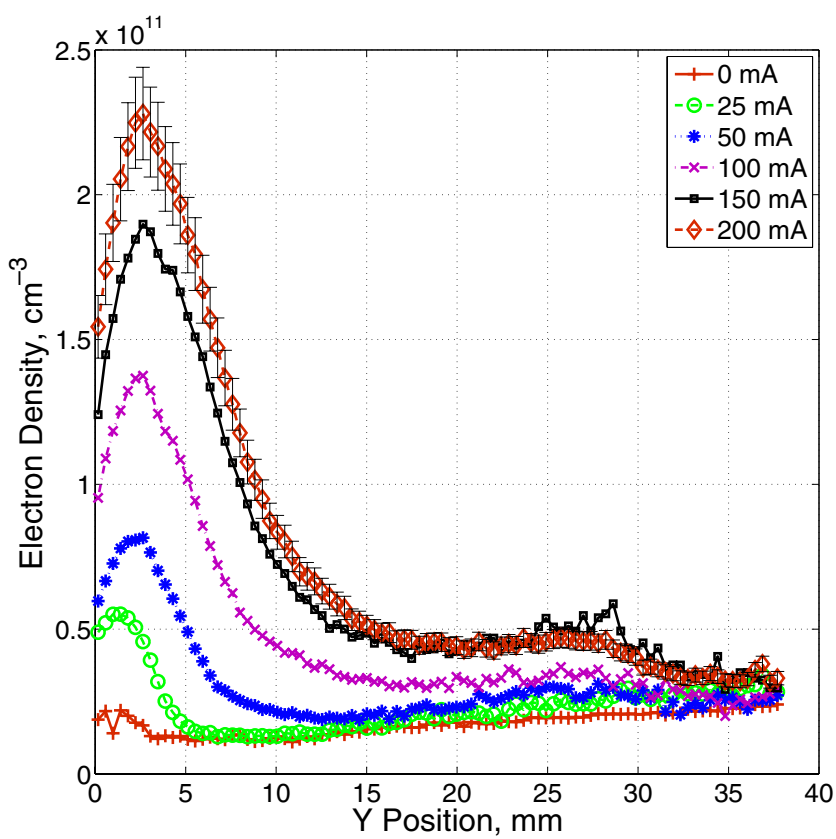

Figure 6. Electron density along the $y$-axis, with $6.4 \mathrm{~mm}$ aperture, at $200 \mathrm{mTorr}$. The aperture is located at $y=0 \mathrm{~mm}$. Error bars show relative uncertainty in electron density.

from this assumption. Temperatures shown in these images are therefore denoted as 'effective' temperatures, which qualitatively indicate the presence of high-energy electron populations relative to lower-energy regions. 


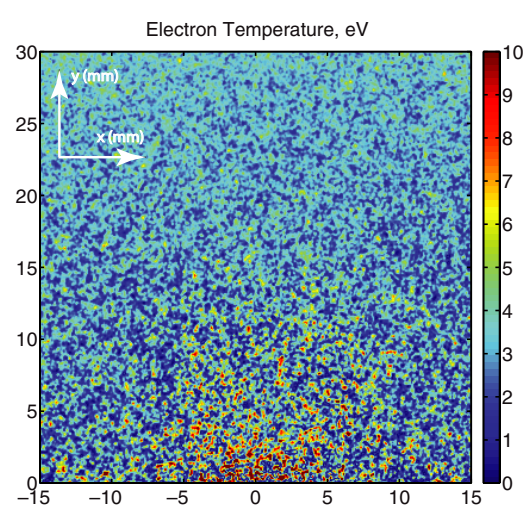

(a) $0 \mathrm{~mA}$

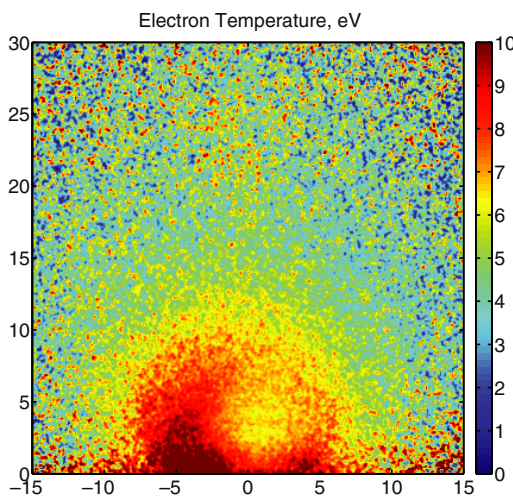

(d) $100 \mathrm{~mA}$

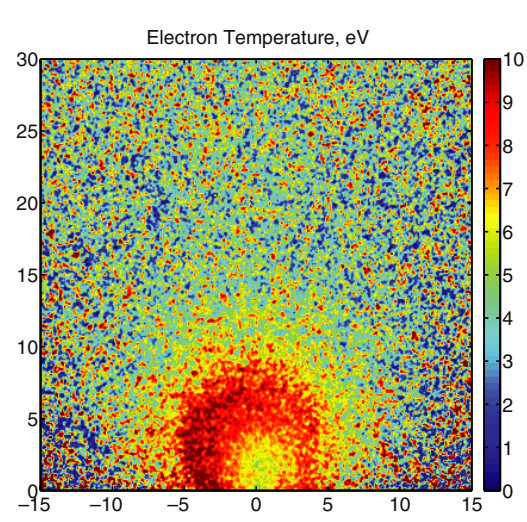

(b) $25 \mathrm{~mA}$

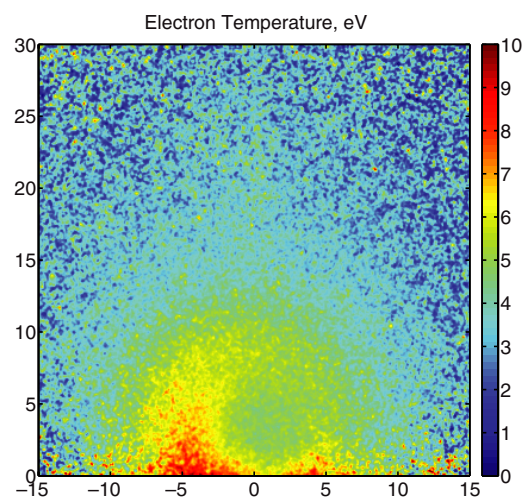

(e) $150 \mathrm{~mA}$

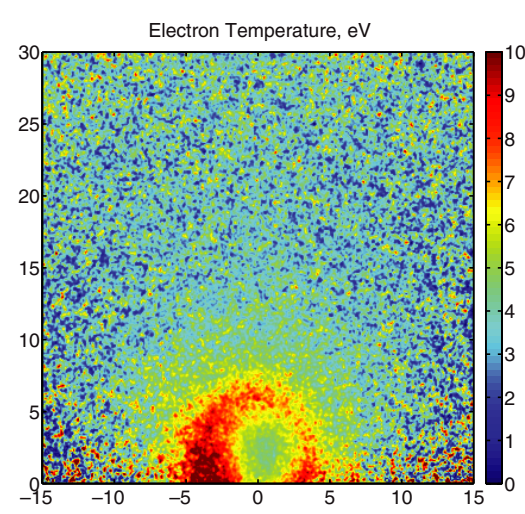

(c) $50 \mathrm{~mA}$

Figure 7. Maps of effective electron temperature with $6.4 \mathrm{~mm}$ aperture, $130 \mathrm{mTorr}$. Axis labels denote position, in $\mathrm{mm}$.

\subsection{Current versus voltage characteristics}

The steady-state current-voltage characteristics of the plasma cathode are shown for pressures of 100 and 200 mTorr in figure 9, with the direction of the voltage sweep during these measurements displayed by arrows on each curve. These curves were generated starting at zero voltage, increasing the voltage (manually, by roughly $1 \mathrm{~V} \mathrm{~s}^{-1}$ ) until the structure was observed. The voltage was held constant for $\sim 5 \mathrm{~s}$ and the dc current drawn by the power supply was recorded. This procedure was repeated at each point as the voltage was gradually increased to $90-100 \mathrm{~V}$, and then decreased until the structure was extinguished. In the initial regime (in which no aperture plasma was present), the collected current was $\sim 1-$ $2 \mathrm{~mA}$; this section of the curve is not shown in figure 9 for clarity. As the bias was increased beyond a threshold value, defined as the 'critical bias,' the aperture plasma formed and the collected current increased dramatically. The critical bias appears in figure 9 as points intercepting the horizontal axis. Once the aperture plasma is formed, the extracted current continues to increase with voltage at a decreasing rate, and at high voltage, the extracted current tends to saturate. Once the aperture plasma was established, the anode bias could be reduced to levels below the critical bias without the extinction of the aperture plasma. The extracted current continued to decrease along with the bias in this regime, until the bias was reduced below an extinction voltage, shown as the endpoint of the $I-V$ curves in figure 9 . The maximum extractable current increased by roughly $20 \%$ as the pressure was decreased from 200 to 100 mTorr. While the internal plasma density did not change appreciably between the two pressures, the conductivity across the aperture-anode gap decreases with pressure. The dependence of peak current on pressure is likely due to the competition of decreasing conductivity and a slightly larger aperture plasma size at 200 mTorr, as discussed in more detail later.

One-dimensional profiles of the electron densities at 130 mTorr, on the voltage downswing starting at $90 \mathrm{~V}$, are shown in figure 10. After the aperture plasma structure was initiated, the anode bias was increased from $80 \mathrm{~V}$, where the extracted current was $262 \mathrm{~mA}$, to $90 \mathrm{~V}$, where the current reached a plateau around $275 \mathrm{~mA}$. The anode bias was then decreased and images were taken at 80, 70, 60 and $55 \mathrm{~V}$. At $55 \mathrm{~V}$, the aperture plasma structure was on the verge of the aperture plasma extinction, and the structure had contracted to nearly the size of the aperture itself. The extracted current for this image was $70 \mathrm{~mA}$, and the aperture plasma was extinguished when the bias was decreased further, in agreement with the data in figure 9.

\subsection{Pressure and aperture size}

One-dimensional density profiles taken at various pressures and a fixed anode bias of $80 \mathrm{~V}$ are shown in figure 11. Peak electron densities vary from 2 to $3 \times 10^{11} \mathrm{~cm}^{-3}$ over a pressure range from 100 to 200 mTorr. At fixed voltage, the electron density profiles have similar spherical shapes over this range of 


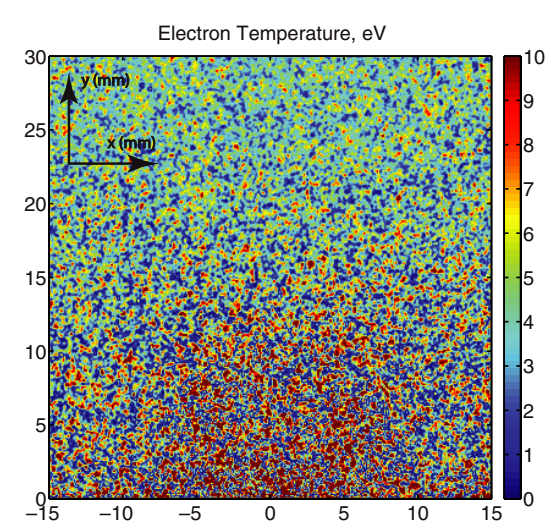

(a) $0 \mathrm{~mA}$

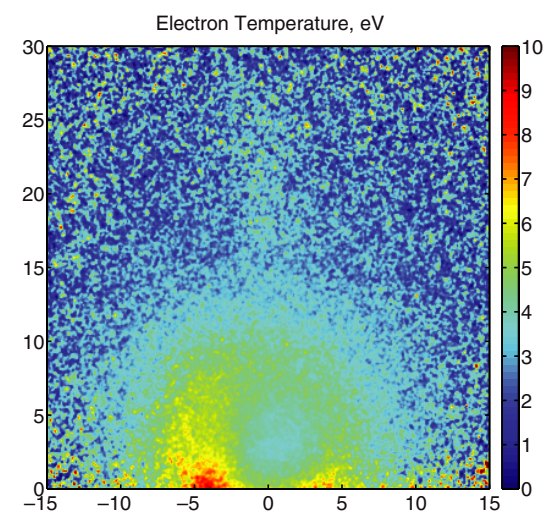

(d) $100 \mathrm{~mA}$

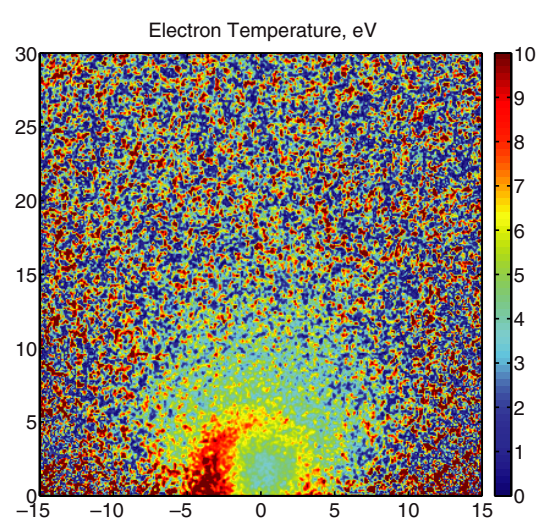

(b) $25 \mathrm{~mA}$

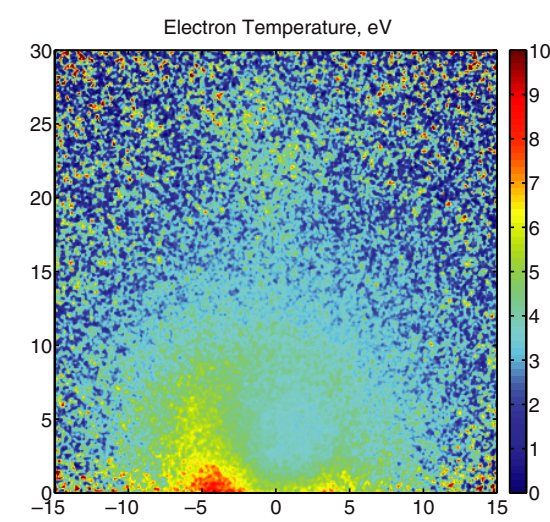

(e) $150 \mathrm{~mA}$

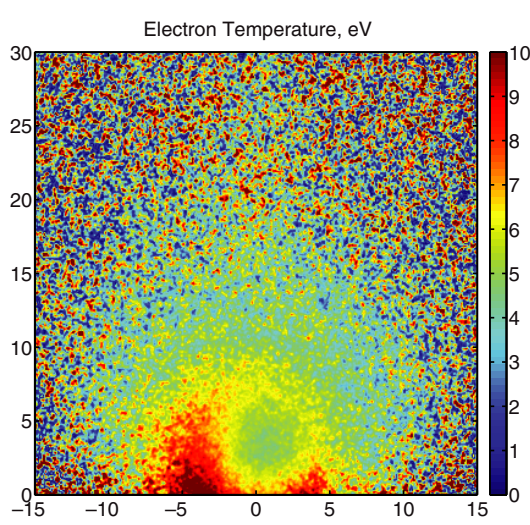

(c) $50 \mathrm{~mA}$

Figure 8. Maps of effective electron temperature with $6.4 \mathrm{~mm}$ aperture, $200 \mathrm{mTorr}$. Axis labels denote position, in mm.

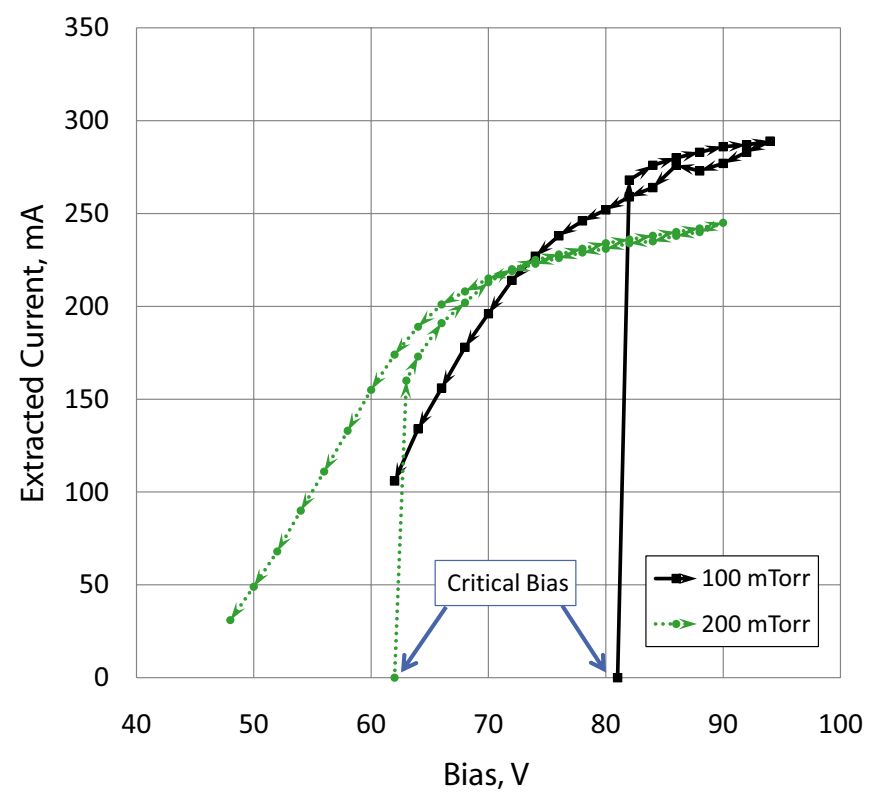

Figure 9. Extracted current versus voltage characteristic, with $6.4 \mathrm{~mm}$ aperture.

pressures. Generally, the dependence of the peak density and structure size on pressure is not as strong as the dependence on extracted current or anode bias, as shown in figures 6 and 10. At elevated pressures, there is a slightly higher electron density in the bulk plasma upstream of the aperture plasma: $\sim 7 \times 10^{10} \mathrm{~cm}^{-3}$ at 200 mTorr, relative to $\sim 5 \times 10^{10} \mathrm{~cm}^{-3}$ at 100 mTorr.

Images of electron density are shown with variable aperture size, fixed pressure and fixed anode current in figure 12 . The case with no aperture plate shows the density with the entire $25 \mathrm{~mm}$ tube open, and the extraction anode exposed. In this aperture-free case, there is a slight increase in density relative to the case with zero bias, but in the presence of the aperture, the plasma structure is clearly prominent. The smallest aperture produces a rounded shape that extends further from the aperture and reaches a peak density that is $60 \%$ higher than with the two larger apertures. To collect a given electron current at the anode, a higher current density is required at the aperture, so the required electric field at the aperture is higher as well. The higher anode bias with the smaller aperture forces the aperture plasma structure to expand outward, producing a larger spot. With the largest aperture, a relatively small structure is formed, with a shape that is more cylindrical than spherical. The formation of a double layer near the aperture plasma arises from the balance of electron and ion current losses from the bulk plasma. In cases where the electron loss area is sufficiently large, elongated structures will form in order to reduce the loss area for electrons while still maintaining a monotonically decreasing potential across the boundary of the spot. The one-dimensional density profiles are shown in figure 13, giving peak densities of $1.5 \times 10^{11} \mathrm{~cm}^{-3}$ for the 7.9 and $6.4 \mathrm{~mm}$ diameter apertures and $2.5 \times 10^{11} \mathrm{~cm}^{-3}$ for the $4.8 \mathrm{~mm}$ aperture. 


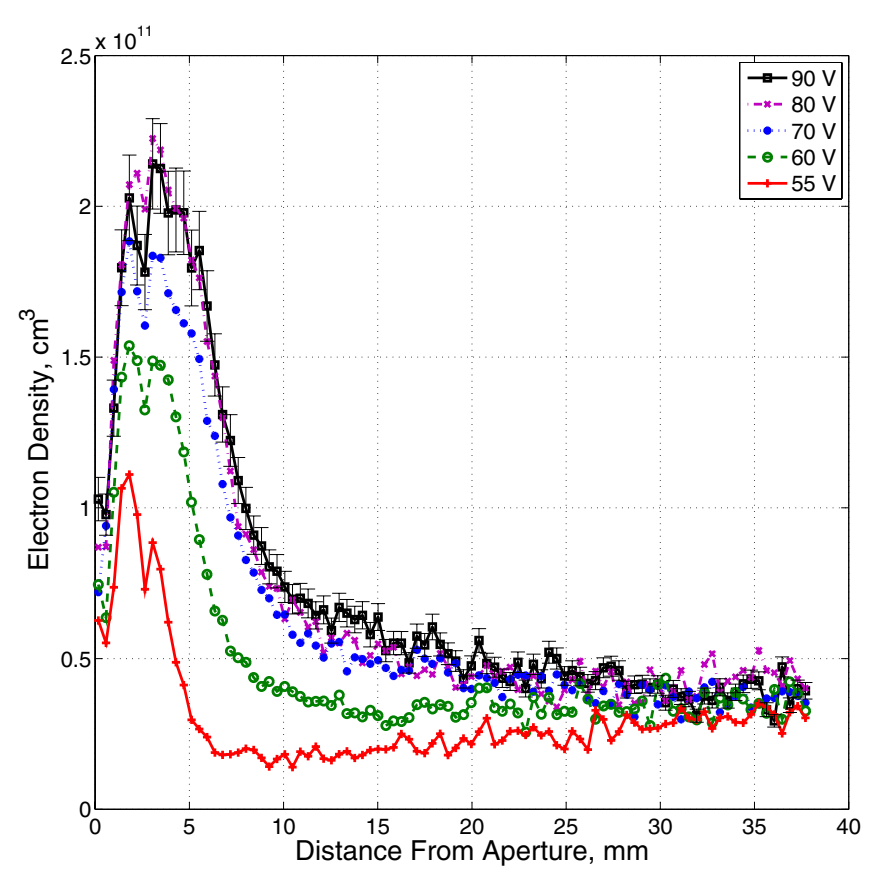

Figure 10. Electron density profiles along the $y$-axis during voltage sweep, with $6.4 \mathrm{~mm}$ aperture, at $130 \mathrm{mTorr}$. The aperture is located at $y=0 \mathrm{~mm}$. Error bars show relative uncertainty in electron density.

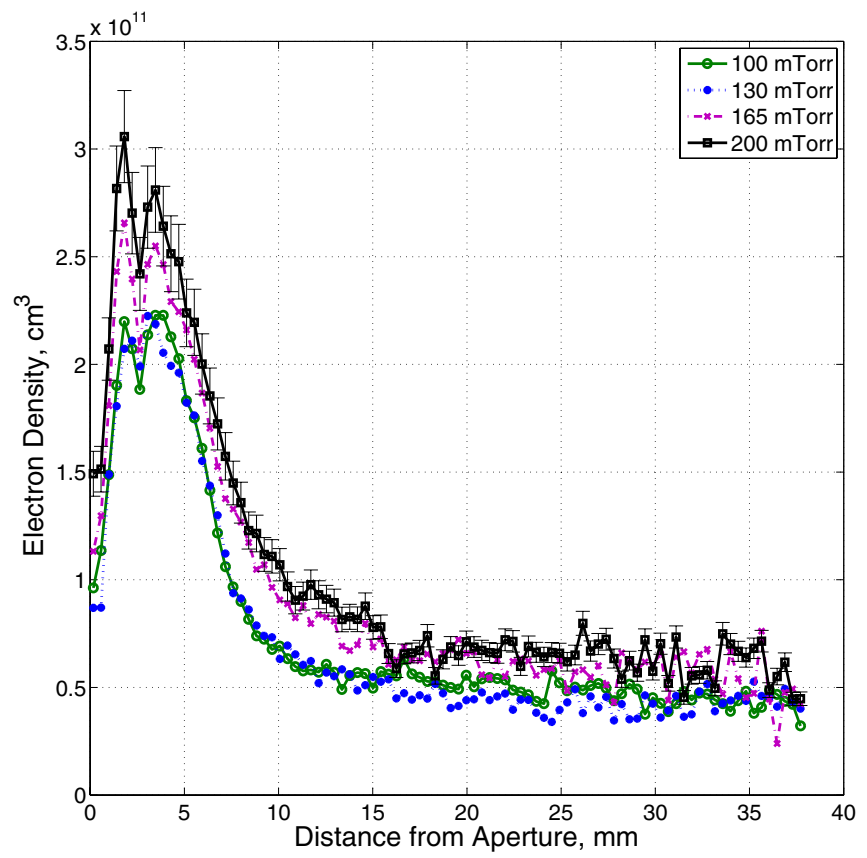

Figure 11. Electron density profiles along the $y$-axis at variable pressure, with $6.4 \mathrm{~mm}$ aperture, at $80 \mathrm{~V}$. The aperture is located at $y=0 \mathrm{~mm}$. Error bars show relative uncertainty in electron density.

\subsection{Plasma potential measurements}

Two sets of Langmuir probe traces were used to quantify the difference in plasma potential between the aperture and bulk plasmas, for two orifice sizes and at two pressures, as a function of extracted current. One probe was centered in the aperture plasma structure, $3 \mathrm{~mm}$ above the aperture; the other probe was $25 \mathrm{~mm}$ above the aperture, in the bulk plasma. The difference in plasma potential between the two probe locations was calculated, and the results are shown in figure 14. With no current extraction (that is, zero anode bias), the plasma potential at the two locations are equal, as expected since no aperture plasma structure is formed. In all other cases, a potential drop on the order of 20-40 V exists between the two probes, supporting the possibility of a double layer sheath at the outer boundary of the structure. This potential drop would focus electrons toward the aperture and drive an increase in ionization in the aperture region. This mechanism is similar to that described in [15], wherein anode spots are formed near a surface (in that case, an anode) which collects only electrons from a quasineutral bulk plasma. The key difference here is that the aperture acts as the loss area adjacent to the bulk plasma; but similarly, all electrons that pass through the aperture area are likely lost to the external anode.

\section{Analysis and discussion}

\subsection{Current-voltage characteristics}

The current-voltage characteristics of the plasma cathode setup, presented in figure 9, are similar to those observed in anode spot studies at lower pressures $[15,33,34]$. One recent explanation for the critical bias and hysteresis behavior in anode spots suggests that the requirement for anode spot onset is that the number densities of ions and electrons in a Debye cube in the spot must be equal, establishing quasineutrality [15]. Once this condition is met, ions born within the spot must enter the bulk plasma with a speed at or exceeding the ion sound speed, in order to satisfy the Bohm criterion for a stable sheath. As a consequence, a quasineutral presheath several Debye lengths in size must form within the anode spot, and the double layer is pushed outward from the anode. On the voltage upswing, the only source of ionization is within the thin anode glow at the electrode surface. After the anode spot is established, the spot itself acts as an additional source of ions (along with the anode glow), and the spot can be sustained at lower voltages.

These same trends are observed in the plasma cathode setup, but in this case, the 'anode spot' is formed upstream of the aperture, far removed from the actual anode surface. Instead, the aperture itself acts as the loss surface for electrons, which produce trace ionization as they are extracted from the plasma cathode. Before spot formation, trace ionization occurs downstream of the aperture as electrons are extracted and accelerated from the source plasma. At high anode voltages, this trace ionization can build up to the point where the quasineutral condition is satisfied; a similar type of 'spot' forms upstream of the aperture in order to maintain a stable sheath for ions produced downstream to be lost to the bulk plasma.

The similarity between the aperture plasma structure and anode spots is clear in the dependence of the critical bias on aperture size and pressure, shown in figure 15. At all pressures, the smaller the aperture, the higher the required voltage for aperture plasma formation. This behavior may be expected, because with smaller electron loss areas, a higher degree of 


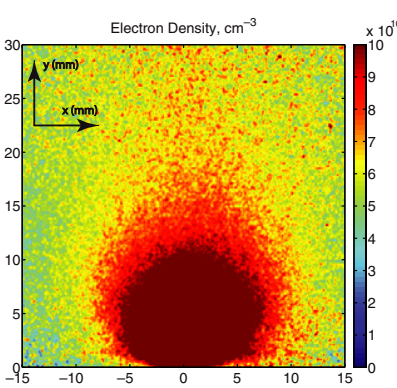

(a) $4.8 \mathrm{~mm}$

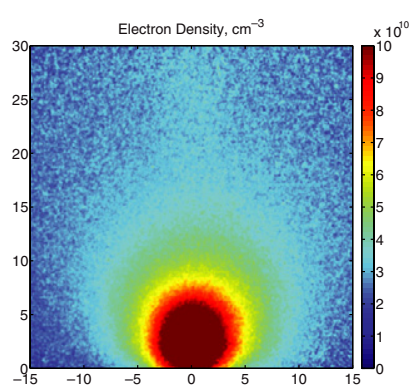

(b) $6.4 \mathrm{~mm}$

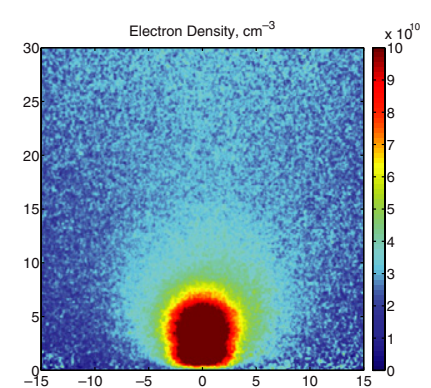

(a) $7.9 \mathrm{~mm}$

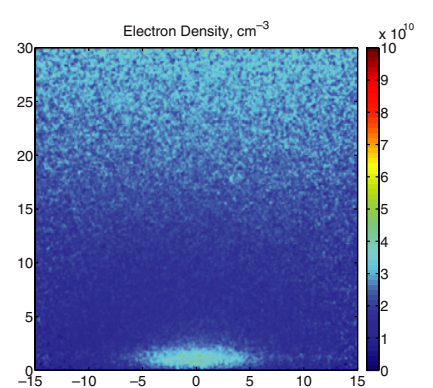

(a) Open

Figure 12. Maps of electron density with variable aperture size, at $150 \mathrm{~mA}$ and $130 \mathrm{mTorr}$. Axis labels denote position, in mm.

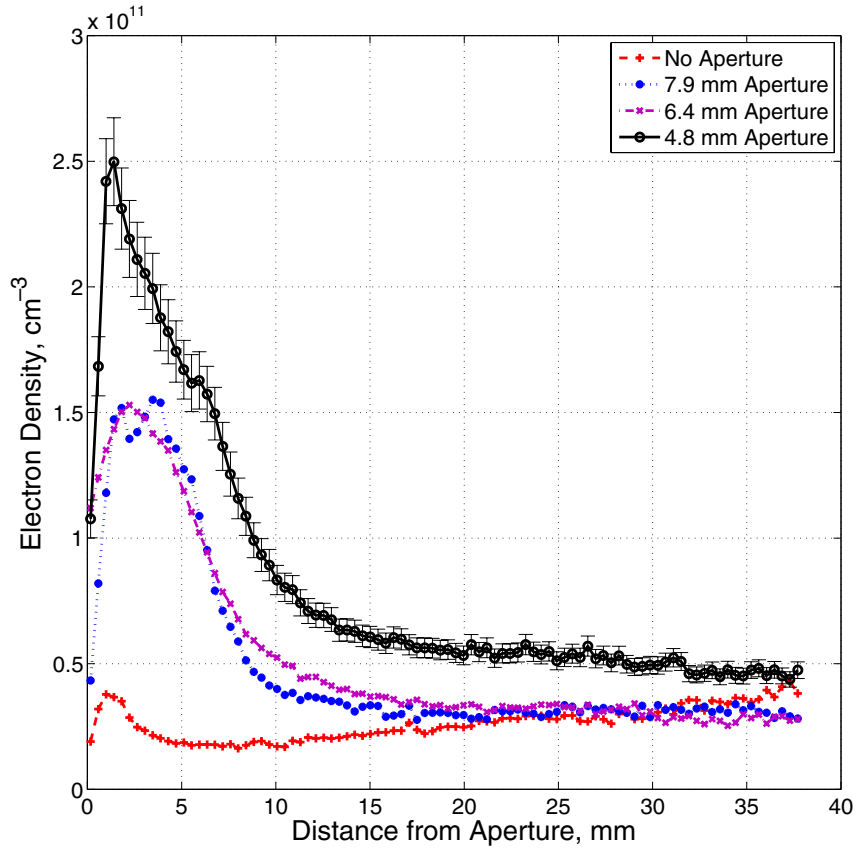

Figure 13. Electron density profiles along the $y$-axis with four aperture configurations; at $150 \mathrm{~mA}$ and $130 \mathrm{mTorr}$. The aperture is located at $y=0 \mathrm{~mm}$. Error bars show relative uncertainty in electron density.

ionization within the aperture plasma is needed to sustain the spot. With small apertures, higher plasma densities are required to supply a given total current. A high anode bias will expand the aperture plasma boundary, accelerating more electrons from the bulk plasma toward the aperture, increasing ionization in that region. Perhaps more interesting is the dependence of the critical bias on pressure, where in figure 15 , the critical bias is shown to scale linearly with $1 / P$ in the low pressure limit. These trends are consistent with anode spot studies at lower pressures, wherein the critical bias was shown to scale linearly with $1 / P[15,33]$. This scaling arises from the dependence of the ionization rate in the plasma spot on the neutral pressure. As the neutral pressure increases, the sheath potential drop required to sustain the minimum ion density for spot formation is decreased. At higher pressures, the critical bias appears to deviate from the $1 / P$ scaling and varies weakly with pressure, and this deviation from the linear scaling is much more pronounced when using a smaller aperture. It may be that in the case of a small aperture, the applied electric field

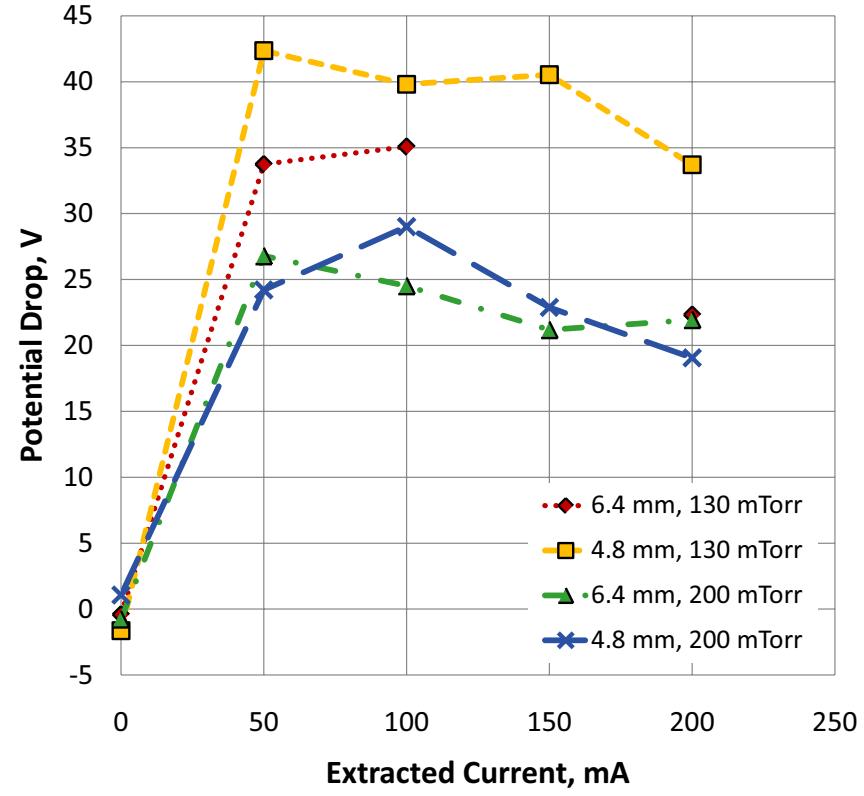

Figure 14. Difference in plasma potential between the bulk and aperture plasmas.

is partially shielded by the aperture; that is, the penetration of even a vacuum electric field into the source plasma chamber is limited by a small aperture. Therefore, at low pressures, the required electric field to sustain the spot can require higher voltages relative to the larger aperture sizes. In the opposite extreme, the 'no aperture' case where the plasma cathode is open to the extraction anode, the scaling is linear with $1 / P$ over the entire pressure range; this is expected since this configuration most closely resembles the anode spot studies in the literature.

\subsection{Aperture plasma size and density}

The electron density maps, current-voltage characteristics and probe measurements showing a large change in potential between the aperture and bulk plasmas are suggestive of a possible double layer sheath at the aperture plasma boundary. If such a sheath exists, then the outer boundary of the structure would collect electrons from the bulk plasma and accelerate them inward toward the aperture, creating a high-density region at the aperture from which high current densities can be extracted. The relationship between the size of this structure, 


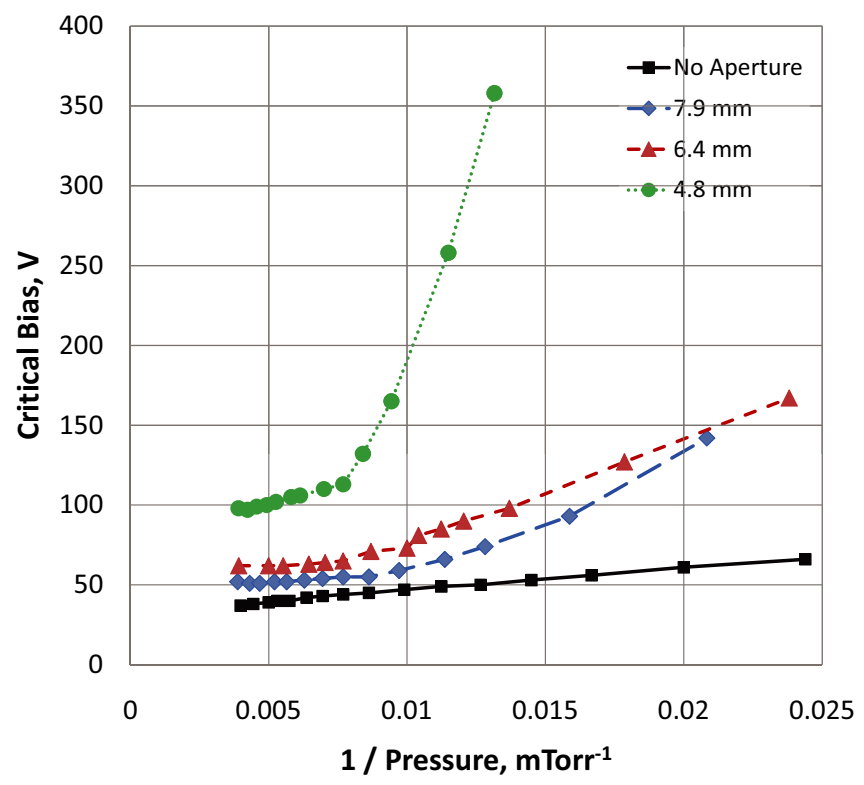

Figure 15. Critical bias for aperture plasma initiation, as a function of pressure, with varying aperture diameters.

the resulting plasma density at the extraction aperture and the extracted current must be considered in the design of plasma cathode electron sources. Here, the results of the LCIF and probe measurements are discussed in the context of this important relationship.

Electrons born within the aperture plasma are not expected to contribute significantly to the extracted current, because they must be balanced by ion losses to the bulk plasma. The maximum ion current density from the aperture plasma to the bulk plasma is limited by the Bohm current, which is a fraction of the extractable electron current density roughly equal to $\left(2 \pi M_{\mathrm{e}} / M_{\mathrm{i}}\right)^{1 / 2}$. Instead, the dependence of the collected current on the applied bias is likely due to the expansion and contraction of the aperture plasma as the voltage is varied. As the voltage is decreased, the aperture plasma surface area contracts. The maximum electron current density that can be extracted from the bulk plasma is limited by the electron saturation current; as the surface area decreases, the current must decrease correspondingly. The radius of the aperture plasma structure (defined as the FWHM of the onedimensional density profile) is plotted against the anode bias in figure 16 for the $6.4 \mathrm{~mm}$ aperture. Figure 16 shows that the radius increases with applied bias, up to a voltage where the radius approaches a maximum $(>70 \mathrm{~V})$. The saturation of the current-voltage characteristics at high voltage (shown in figure 9) is likely due to the aperture plasma reaching its maximum surface area, limiting the extracted current.

With the $6.4 \mathrm{~mm}$ aperture at $200 \mathrm{mTorr}$, the bulk density converges to the same value at a few centimeters from the aperture as the extracted current is varied (figure 6). At low current levels, there appears to be a dip in the density between the bulk and aperture plasmas. This may be a result of rarefaction of the incoming electron 'beam' as it is accelerated through the double layer toward the aperture plasma. As the electrons are accelerated, if flux is roughly conserved across the double layer, the density will decrease correspondingly. At

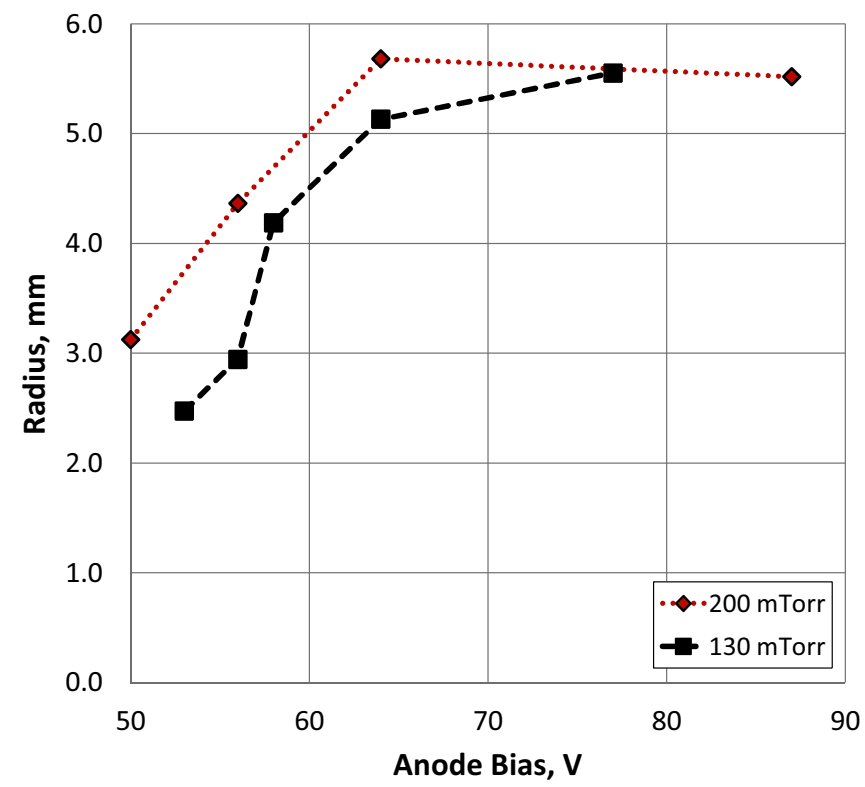

Figure 16. Radius of aperture plasma structure as a function of anode bias, with $6.4 \mathrm{~mm}$ aperture.

higher currents, the emergence of a second 'bump' in electron density starts to form roughly $2 \mathrm{~cm}$ upstream of the density peak. Ions produced within the aperture plasma will see an outward acceleration by the double layer sheath. As these ions are accelerated away from they aperture, they may undergo charge exchange collisions, resulting in a region that is ionrich to a small extent. This region in an otherwise quasineutral plasma will tend to attract electrons, and a small, diffuse peak in plasma density can form via the influx of expelled ions balancing losses from ambipolar diffusion.

The size and shape of the aperture plasma depends on aperture size as well. In particular, the smallest aperture investigated in this work $(4.8 \mathrm{~mm})$ was associated with the largest plasma structure. In order to maintain a constant current, the smaller aperture requires a higher current density, and therefore a higher electron density at the aperture. To increase the electron density at the aperture, the outer boundary of the structure expands in order to increase the collection area for bulk electrons. A larger number of collected electrons are then focused toward the aperture by the potential drop, resulting in a higher electron density at the aperture. With the larger $(7.9 \mathrm{~mm})$ aperture, the smaller plasma spot for a fixed current (relative to the case with the smaller aperture) is likely associated with a larger physical extraction opening and a lower current density at the aperture. The boundary of the structure can be cylindrical in this case to reduce the interface area between the aperture plasma structure and the source plasma, while still supplying the necessary current density at the aperture.

The electron density on the upstream side of the aperture may be estimated from the extracted current, gas pressure and plasma cathode geometry as follows. With the assumption that trace ionization downstream of the aperture does not significantly multiply the collected current (a valid assumption since the downstream region is surrounded by insulating material), the total current collected equals the current through 


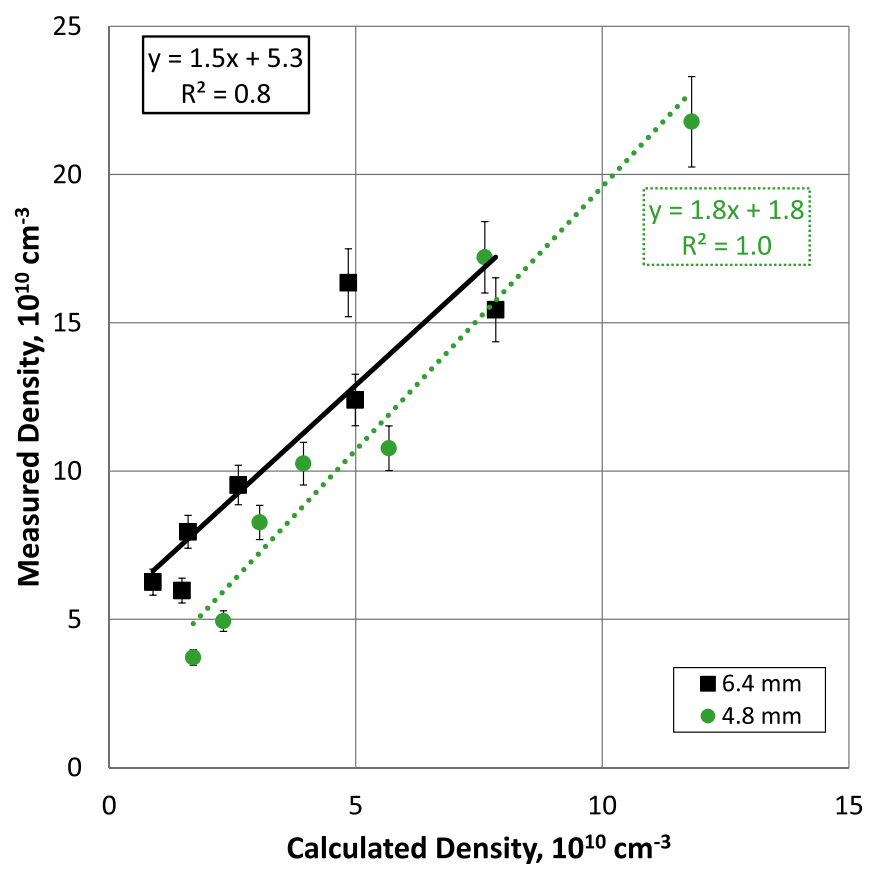

Figure 17. Measured versus calculated electron density, with 6.4 and $4.8 \mathrm{~mm}$ apertures. Error bars show relative uncertainty due to the LCIF analysis.

the aperture itself. If the sheath thickness around the aperture plate is small relative to the aperture diameter, the collected current is

$$
I_{\mathrm{ext}}=J_{\mathrm{ap}} A_{\mathrm{ap}}=e n_{\mathrm{ap}} v_{\mathrm{d}}(E / N) \pi d_{\mathrm{ap}}^{2} / 4,
$$

where $I_{\mathrm{ext}}$ is the total extracted current, $J_{\mathrm{ap}}$ is the current density through the aperture, $A_{\text {ap }}$ is the area of the aperture $n_{\text {ap }}$ is the electron density at the aperture and $v_{\mathrm{d}}$ is the electron drift velocity, which is a function of the reduced electric field, $E / N$. The potential change experienced by extracted electrons is estimated as the difference in the anode bias, $V_{\text {bias }}$ and corresponding bulk plasma potential in steady state, $V_{\text {bulk }}$. The value of $E / N$ in the gap is estimated from

$$
E / N \approx \frac{V_{\text {bias }}-V_{\text {bulk }}}{l N_{\mathrm{g}}},
$$

where $l$ is the length of the anode-aperture gap and $N_{\mathrm{g}}$ is the neutral gas density. Values of $V_{\text {bulk }}$ were taken from the Langmuir probe traces, and the electron drift velocity under each condition was estimated from $E / N$ from the data in [35]. The electron density at the aperture acquired from LCIF was plotted against the calculated aperture plasma density $\left(n_{\text {ap }}\right.$ in equation (4)), and the results are shown in figure 17 for combined measurements at 130 and 200 mTorr with the 6.4 and $4.6 \mathrm{~mm}$ diameter apertures.

For both aperture sizes, there is a clear linear relationship between the calculated and measured electron densities. The calculation underestimates the electron density by $50 \%$ for the larger aperture and $80 \%$ for the smaller aperture; a factor of $50 \%$ is within the systematic uncertainty of the LCIF measurement. The linear fit with the $4.8 \mathrm{~mm}$ aperture is clear, and the measured density intercepts the vertical axis at the bulk plasma density, as expected. The trend with the $6.4 \mathrm{~mm}$ aperture is less defined; but as the aperture size increases, more electric field leakage into the source plasma region is expected, and the 1D model may fail. An analysis including two-dimensional variations in the field on the upstream side of the aperture would require a more detailed model outside the scope of this mainly experimental work.

\subsection{Electron current into aperture plasma}

The LCIF density profiles were also used to study the relationship between electron flux from the source plasma and the extracted current with all three aperture sizes. The Maxwellian electron flux entering the aperture plasma from the bulk was calculated from the electron density profiles and comparing this with the extracted current. The electron saturation current density, $J_{\text {sat }}$, was calculated according to

$$
J_{\mathrm{sat}}=\frac{e n}{4} \sqrt{\frac{8 k_{\mathrm{B}} T_{\mathrm{e}}}{\pi M_{\mathrm{e}}}} .
$$

The shape of the aperture plasma was assumed to be an ellipsoid with axes defined as the FWHM relative to the peak plasma density, in the horizontal and vertical directions. The selection of the FWHM is admittedly arbitrary, since an accurate definition of the boundary would be the mid-point of the double layer potential drop around the aperture plasma. Another estimate of the boundary may be the location of the maximum gradient in electron density; this was generally within $0.5 \mathrm{~mm}$ of the FWHM location. The interface boundary area was defined as the portion of the ellipsoid in contact with the bulk plasma, i.e. the ellipsoid area on the upstream side of the aperture. The current density times the boundary area was integrated around the boundary,

$$
I_{\text {bulk }}=\int_{A} J \cdot \mathrm{d} A
$$

to give the total available electron current from the bulk, $I_{\text {bulk }}$. The values of electron density and temperature used in the calculation were $2 \times 10^{10} \mathrm{~cm}^{-3}$ and $3 \mathrm{eV}$, respectively, corresponding to the properties of the bulk plasma. This total current was plotted against the extracted current for all three aperture sizes, as shown in figure 18.

In all cases, the extracted current was a fraction of the calculated available current. The calculated current had a strong linear dependence on the extracted current in all cases, though the ratio between the two became closer to unity as the aperture size was increased. One possible contributing factor to the discrepancy between current values is the fact that at 130 mTorr, the elastic scattering mean free path in helium $\left(\lambda_{\mathrm{el}}=0.05 \mathrm{~cm}\right.$, assuming a cross-section of $\left.5 \times 10^{-15} \mathrm{~cm}^{2}\right)$ is much shorter than the ionization path length. Indeed, the scattering path length is smaller than the aperture plasma dimensions. Even if it is assumed that the electron energy distribution function is a monoenergetic $\sim 35 \mathrm{eV}$ beam created by the double layer, then the ionization path length is $\lambda_{\text {in }}=18$ $\mathrm{cm}$, which is still much larger than the aperture plasma structure (assuming an ionization cross-section of $1.29 \times 10^{-17} \mathrm{~cm}^{2}$ 


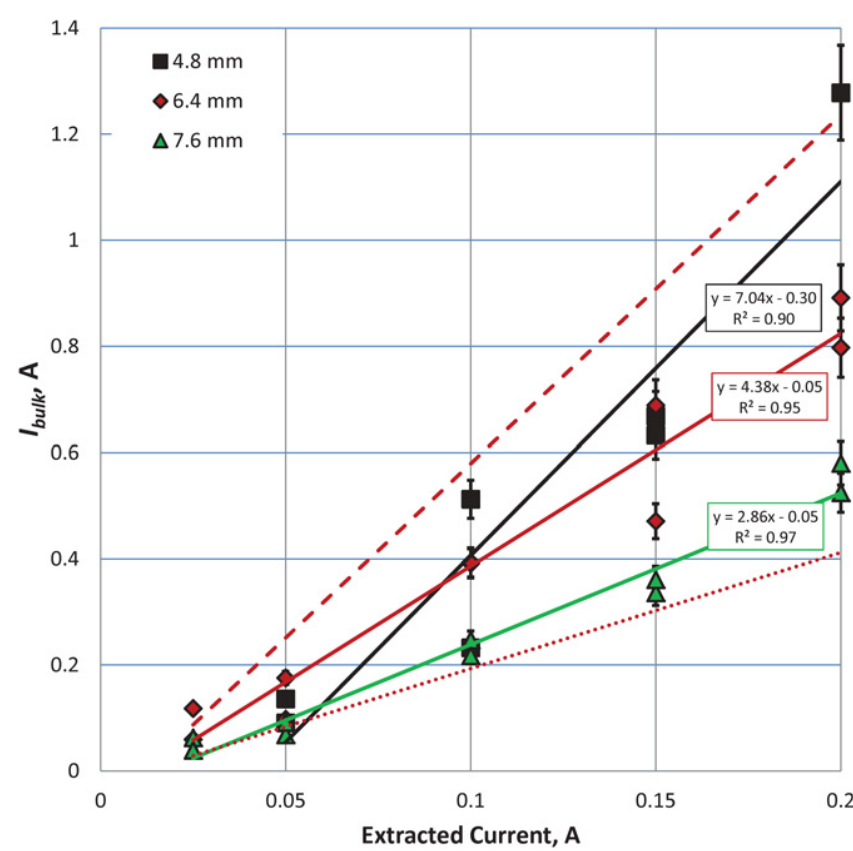

Figure 18. Electron saturation current at aperture plasma boundary versus extracted current, at $130 \mathrm{mTorr}$. Dashed and dotted red trendlines denote range of densities due to systematic uncertainty in LCIF analysis with $6.4 \mathrm{~mm}$ aperture. Error bars show relative uncertainty in electron density.

from [36]). Some of the available electron current at the aperture plasma boundary may not be utilized as extracted current; if each electron is expected to scatter several times within the aperture plasma, while the probability of an inelastic event is low, then it could enter the aperture plasma and scatter back into the bulk plasma before being lost after an inelastic event. The exception would be electrons that are scattered toward the aperture itself, or electrons born near the aperture. The latter must be balanced by a flux of backstreaming ions, however, so that contribution is relatively low. Again, because the ion flux out of the aperture plasma boundary is lower than the electron flux by a factor of $\sqrt{2 \pi M_{\mathrm{e}} / M_{\mathrm{i}}}$, the contribution from ionization in the spot is only a few per cent of the total current.

An estimate of the incident electron flux that is scattered back into the bulk plasma can be estimated with a simple 1D analysis. Let there be an incident electron flux from the bulk, with the boundary between the bulk and aperture defined as $z=0$. Let $\mathrm{d} f_{\text {ref }}$ be the fraction of incident electrons which reach a depth $x$ without collision, are scattered backward in the next differential distance $\mathrm{d} x$, and escape from the spot by again traversing the distance $x$ without scattering. The differential fraction of reflected electrons can be written as

$$
\mathrm{d} f_{\text {ref }}=f_{\text {inc }} \times \mathrm{d} f_{\text {coll }} \times f_{\text {esc }},
$$

where $f_{\text {inc }}$ is the fraction that reach depth $x, \mathrm{~d} f_{\text {coll }}$ is the fraction of these that are scattered backward in $\mathrm{d} x$ and $f_{\text {esc }}$ is the fraction of backward scattered electrons that escape without a second collision. The quantities $f_{\text {inc }}$ and $f_{\text {esc }}$ are given by

$$
f_{\text {inc }}=f_{\text {esc }}=\mathrm{e}^{-x / \lambda_{\mathrm{e}}} \text {, }
$$

where $\lambda_{\mathrm{e}}$ is the elastic scattering mean free path. Assuming isotropic scattering, the quantity $\mathrm{d} f_{\text {coll }}$ is

$$
\mathrm{d} f_{\text {coll }}=\frac{1}{2} N_{\mathrm{g}} \sigma_{\mathrm{e}} \mathrm{d} x=\frac{\mathrm{d} x}{2 \lambda_{\mathrm{e}}},
$$

where $\sigma_{\mathrm{e}}$ is the elastic scattering cross-section. For spot sizes much larger than the scattering pathlength, the lower bound on the reflected electron fraction from single collisions is found by combining equations (8)-(10) and integrating over all $x$; this yields a value of $f_{\text {ref }}=1 / 4$. This includes singlescatter events only, but in reality an electron can scatter several times without an inelastic event and return to the bulk plasma. In the extreme limit of this case, the fraction of electrons returned to the bulk plasma may be estimated as the ratio of the spot-bulk interface area to the total (spot + aperture) area. In the case of the $6.4 \mathrm{~mm}$ diameter aperture at $130 \mathrm{mTorr}$ and $100 \mathrm{~mA}$, for instance, the average spot radius is roughly $5 \mathrm{~mm}$, so the upper bound of the reflected fraction would be $\sim\left(4 \times 5^{2}-3.2^{2}\right) /\left(4 \times 5^{2}\right) \approx 0.9$. At this pressure, the mean drift distance traveled between inelastic collisions is $\lambda_{\mathrm{t}}=\sqrt{\lambda_{\mathrm{el}} \lambda_{\text {in }}} \approx 1 \mathrm{~mm}$, less than the aperture plasma radius; Thus, inelastic events cannot be neglected and the reflection fraction is in an intermediate regime between the two bounds given here. The combination of systematic uncertainty in the LCIF measurement with the minimum reflection fraction of $1 / 4$ could explain up to a factor of $\sim 2$ discrepancy between the extracted current and current collected by the spot. A larger discrepancy, as seen with the smaller apertures, may imply a higher reflection fraction from the bulk due to multiple scattering events before the incoming electrons can 'find' the aperture. With the $4.8 \mathrm{~mm}$ aperture, the electron density profile is shaped more like a teardrop than an ellipsoid, as seen in the 2D LCIF images. The analysis fitting an elliptical boundary to the effective minor and major FWHM of the spot would tend to overestimate the size of the structure, which contributes to the weaker fit seen in figure 18 .

It should be noted that even though the extracted current is smaller than the integrated electron saturation current over the boundary of the structure, the bulk plasma alone is not of a high enough density to deliver the currents shown in figure 9. If electron extraction from a quasineutral plasma were simply limited by the electron saturation current through the aperture, rather than the current into the aperture plasma boundary, the bulk plasma would deliver a current density of $90 \mathrm{~mA} \mathrm{~cm}^{-2}$. With the $6.4 \mathrm{~mm}$ aperture, this translates to $30 \mathrm{~mA}$ of total current, well below the $260 \mathrm{~mA}$ mark that was achieved at 130 mTorr. Similarly, with the $4.8 \mathrm{~mm}$ aperture, the total current would be $16 \mathrm{~mA}$, while up to $200 \mathrm{~mA}$ was measured. The data in this work clearly show that the expanding aperture plasma boundary plays an important role as an expanding effective loss area for electrons as they are extracted from the plasma cathode aperture.

\section{Conclusions}

The development and properties of the aperture plasma structure have important consequences in the design of plasma cathode devices. The structure has been shown to behave in 
similar ways to anode spots at the surface of positively biased electrodes immersed in a quasineutral discharge. However, the density at the aperture itself is dictated by the current extracted via the external electric field applied across the anode-aperture gap. As a consequence, the boundary of the structure expands into the bulk plasma to increase the collected electron flux from the bulk, supporting the necessary current density through the aperture for a given bias applied across the gap. It appears that in plasma cathode design, it may be possible to optimize extraction aperture dimensions to accommodate specific emission requirements. In applications requiring very high feed gas utilization, for example, one could use a small aperture to increase the internal plasma density at a given flow rate, and the aperture plasma boundary can expand to extract high currents from the bulk plasma. There is a tradeoff, however, as the voltage requirements for sustaining the aperture plasma with a smaller aperture can increase. Findings in this work suggest that the aperture plasma structure may be a general property of low temperature plasma cathodes, and is likely necessary, particularly in those cases where high emission is required. More detailed models, which take into account two-dimensional density profiles and extraction electrode geometries, may provide more accurate means of predicting the interrelated nature of current extraction from a plasma, across a large gap, and the response of the internal plasma structure near the interface.

\section{Acknowledgments}

The authors want to thank Ahmed El-Saghir for his insightful work on Langmuir probe experiments that were related to this study. This work was supported by the Department of Energy Office of Fusion Energy Science Contract DE-SC0001939.

\section{References}

[1] Oks E M 1992 Plasma Sources Sci. Technol. $1249-55$

[2] Oks E M and Schanin P M 1999 Phys. Plasmas 6 1649-54

[3] Jameson K, Goebel D and Watkins R 2005 Proc. 41st AIAA/ASME/SAE/ASEE Joint Propulsion Conf. \& Exhibit (Tucson, AZ) AIAA-2005-3667

[4] Goebel D M, Jameson K K, Watkins R M, Katz I and Mikellides I G 2005 J. Appl. Phys. 98113302

[5] Godyak V, Raitses Y and Fisch N J 2007 Proc. 30th Int. Electric Propulsion Conf. (Florence, Italy) IEPC-2007-266

[6] Funaki I, Kuninaka H and Toki K 2004 J. Propulsion Power 20 718-27

[7] Kamhawi H, Foster J E and Patterson M J 2004 Proc. 40th AIAA/ASME/SAE/ASEE Joint Propulsion Conf. and Exhibit (Fort Lauderdale, FL) AIAA-2004-3819
[8] Hidaka Y, Foster J E, Getty W D, Gilgenbach R M and Lau Y Y 2007 J. Vacuum Sci. Technol. A 25 781-90

[9] Weatherford B R and Foster J E 2010 Proc. 46th AIAA/ASME/SAE/ASEE Joint Propulsion Conf. \& Exhibit (Nashville, TN) AIAA-2010-6519

[10] Diamant K D 2009 Proc. 31 st Int. Electric Propulsion Conf. (Ann Arbor, MI) IEPC-2009-015

[11] Diamant K D 2009 IEEE Trans. Plasma Sci. 37 1558-62

[12] Longmier B and Hershkowitz N 2008 Rev. Sci. Instrum. 79093506

[13] Kuninaka H, Nishiyama K, Funaki I, Yamada T, Shimizu Y and Kawaguchi J 2007 J. Propulsion Power 23 544-51

[14] Baalrud S D, Hershkowitz N and Longmier B 2007 Phys. Plasmas 14042109

[15] Baalrud S D, Longmier B and Hershkowitz N 2009 Plasma Sources Sci. Technol. 18035002

[16] Song B, D'Angelo N and Merlino R L 1991 J. Phys. D: Appl. Phys. 24 1789-95

[17] Stenzel R L, Ionita C and Schrittwieser R 2008 Plasma Sources Sci. Technol. 17035006

[18] Tsuchida K, Miyake S, Kadota K and Fujita J 1983 Plasma Phys. 25 991-3

[19] Dubreuil B and Prigent P 1985 J. Phys. B: At. Mol. Phys. 18 4597-611

[20] Den Hartog E A, O'Brian T R and Lawler J E 1989 Phys. Rev. Lett. 62 1500-3

[21] Dzierzȩga K, Musiol K, Benck E C and Roberts J R 1996 J. Appl. Phys. 80 3196-201

[22] Shcheglov D A, Vetrov S I, Moskalenko I V, Skovoroda A A and Shuvaev D A 2006 Plasma Phys. Rep. 32 119-22

[23] Barnat E V and Frederickson K 2010 Plasma Sources Sci. Technol. 19055015

[24] Denkelmann R, Freund S and Maurmann S 2000 Contrib. Plasma Phys. 40 91-5

[25] Ralchenko Y, Janev R K, Kato T, Fursa D V, Bray I and de Heer F J 2008 At. Data Nucl. Data Tables $90603-22$

[26] Godyak V A and Popov O A 1977 Sov. Phys._Tech. Phys. 22461

[27] Weatherford B R, Barnat E V and Foster J E 2011 IEEE Trans. Plasma Sci. 39 2486-7

[28] Stenzel R L, Gruenwald J, Ionita C and Schrittwieser R 2012 Plasma Sources Sci. Technol. 21015012

[29] Stenzel R L, Gruenwald J, Ionita C and Schrittwieser R 2011 Plasma Sources Sci. Technol. 20045017

[30] Stenzel R L, Gruenwald J, Ionita C and Schrittwieser R 2011 Phys. Plasmas 18062112

[31] Stenzel R L, Gruenwald J, Ionita C and Schrittwieser R 2011 Phys. Plasmas 18062113

[32] Mikellides I G, Katz I, Goebel D M and Polk J E 2005 J. Appl. Phys. 98113303

[33] Tang D and Chu P K 2003 J. Appl. Phys. 94 1390-5

[34] Strat M, Strat G and Gurlui S 1999 J. Phys. D: Appl. Phys. 32 34-40

[35] Kücükarpaci H N, Saelee H T and Lucas J 1981 J. Phys. D: Appl. Phys. 14 9-25

[36] Kim Y K and M Eugene Rudd 1994 Phys. Rev. A 503954 